\title{
Caractéristiques énergétiques de cathodes à micropointes à émission de champ
}

\author{
A. Brenac $(*)$, R. Baptist $(* *)$, G. Chauvet $(*)$ et R. Meyer $(* *)$ \\ (*) DRF-IRF, Commissariat à l'Energie Atomique, CEN/G, 85 x, 38041 Grenoble Cedex, France \\ $(* *)$ LETI-IRDI, Commissariat à l'Energie Atomique, CEN/G, 85 x, 38041 Grenoble Cedex, France
}

(Reçu le 5 janvier 1987, révisé le 15 juillet 1987, accepté le 7 septembre 1987)

\begin{abstract}
Résumé. - Dans cet article nous exposons les caractéristiques de cathodes à émission de champ comprenant un grand nombre de pointes de dimensions voisines du micron, regroupées à la surface d'un composant et réalisées par les techniques de la microélectronique. Les résultats théoriques concernant la caractéristique courant-tension sont présentés, ainsi que les propriétés mesurées de cathodes fabriquées au laboratoire : le courant émis à $60 \mathrm{~V}$ par une surface de $1 \mathrm{~mm}^{2}$ comprenant 10000 pointes est environ $100 \mu \mathrm{A}$, la surface émissive par pointe est de quelque $10 \AA^{2}$ et le champ est voisin de $5 \times 10^{9} \mathrm{Vm}^{-1}$. Ensuite nous abordons le problème de la distribution énergétique des électrons émis : les valeurs théoriques attendues, et les méthodes expérimentales utilisées sont présentées. Les mesures mettent en évidence une chute de potentiel qui est attribuée à une résistance d'interface entre les pointes et le substrat sur lequel elles sont déposées (dépendant de la nature du substrat). L'observation de dispersions énergétiques élevées $(2 \mathrm{eV})$ est corrélée à ce phénomène parasite. Pour les cathodes présentant une faible résistance d'interface on a mesuré une dispersion énergétique de $0,9 \mathrm{eV}$, pour un courant de $100 \mu \mathrm{A}$ à $60 \mathrm{eV}$. Il apparaît que les progrès dans ce domaine dépendent de la maîtrise de l'interface. L'observation d'un fort courant, même à très basse température $(<100 \mathrm{~K})$ ouvre le champ d'application de ces cathodes qui pourraient donc servir de sources froides fournissant des faisceaux intenses d'électrons de faible énergie.
\end{abstract}

\begin{abstract}
We present in this paper the characteristics of field emission cathodes consisting of a large number of micron-sized tips, arranged at the surface of a component and realized by using thin film technology. Theoretical results concerning the current-voltage characteristics are presented, as well as the measured properties of cathodes prepared in our laboratory : a current of about $100 \mu \mathrm{A}$ is emitted at $60 \mathrm{~V}$ by a $1 \mathrm{~mm}^{2}$ surface comprising 10000 microtips ; the emitting area is about $10 \AA^{2}$ per tip and the field about $5 \times 10^{9} \mathrm{Vm}^{-1}$. We consider next the energy distribution of the emitted electrons : the expected theoretical results and the experimental methods are presented. The experiment exhibits a voltage drop at the interface between the tips and the substrate on which they are deposited (depending on the nature of the substrate). The observation of a large energetic spread $(2 \mathrm{eV})$ is correlated to this parasitic phenomenon. As for the cathodes which exhibit a low interface resistance, we measured an energetic spread of $0.9 \mathrm{eV}$, at a current of $100 \mu \mathrm{A}$ and an energy of $60 \mathrm{eV}$; it appears that improvements in this field depend on the control of the interface. The emission of high currents, even at a low temperature $(T<100 \mathrm{~K})$, makes it possible to use such cathodes as cold electron sources providing intense beams of low-energy electrons.
\end{abstract}

\section{Introduction.}

Les cathodes équipant les canons à électrons classiques utilisent généralement l'émission thermoélectronique. Leur principe est le suivant : à la surface d'un matériau porté à une température élevée, une fraction des électrons acquiert une énergie thermique supérieure à l'énergie potentielle dans le vide, et peut alors être émise dans le vide, pourvu que la vitesse soit dirigée vers l'extérieur du matériau
(Fig. 1a). La densité de courant émise est donnée par la loi de Dushman-Richardson :

$$
J=A T^{2} \exp \left(-\frac{\phi}{k T}\right)
$$

où $\phi$ est le travail de sortie (pour un conducteur), $T$ la température absolue, et $A$ une constante caractéristique du matériau (de l'ordre de $10^{6} \mathrm{~A} \mathrm{~m}^{-2} \mathrm{~K}^{-2}$ ). Pour que cette densité de courant soit effectivement 


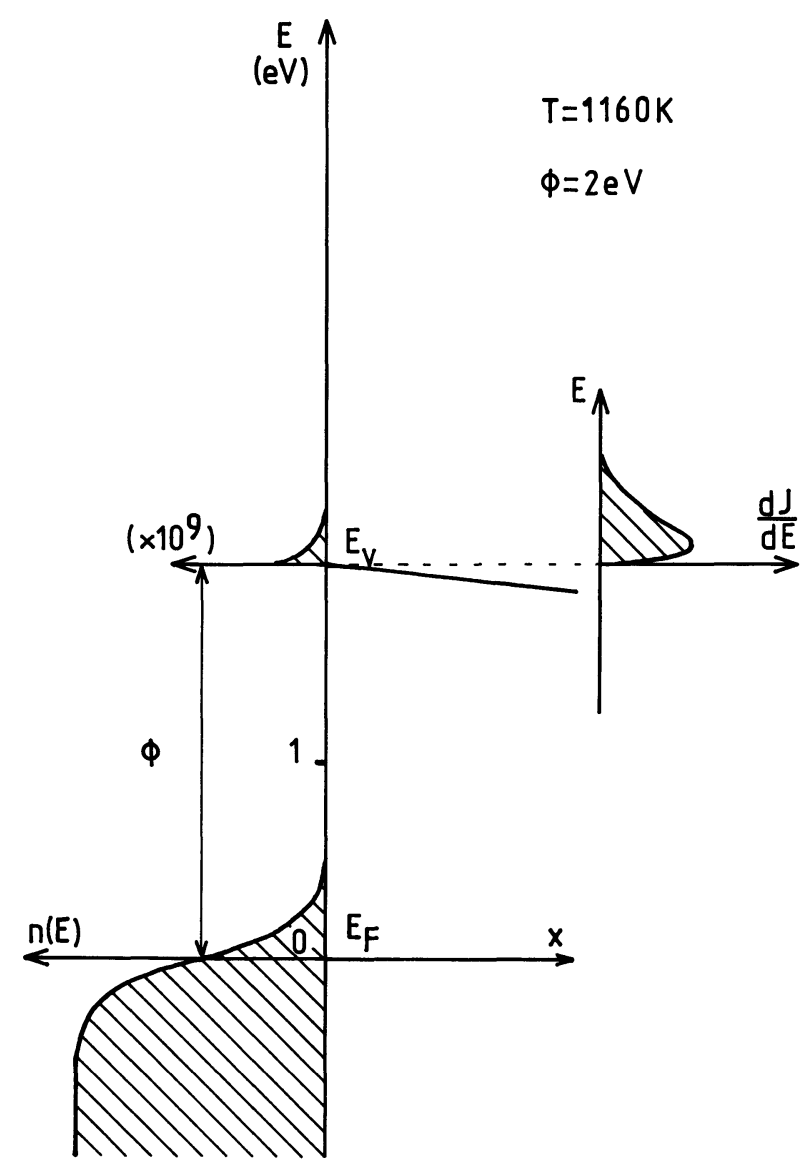

a)

Fig. 1. - Diagramme d'énergie correspondant à l'émission thermoélectronique (1a) et à l'émission de champ (1b).

extraite de la cathode, il faut appliquer un champ à la surface de celle-ci qui puisse «drainer" les électrons. La recherche d'une forte densité de courant conduit à utiliser soit des matériaux réfractaires à haute température, soit des matériaux à faible travail de sortie. Afin de minimiser la dispersion énergétique des électrons émis, qui est donnée par la relation :

$$
\Delta E=2,45 k T
$$

on adopte souvent la seconde solution. Par exemple, pour une cathode "dispenser » à oxyde de baryum $(\phi \simeq 2 \mathrm{eV})$, une température de $880^{\circ} \mathrm{C}$ correspond à une densité de courant de l'ordre de $3 \times 10^{3} \mathrm{~A} \mathrm{~m}^{-2}$ et une dispersion d'environ $0,25 \mathrm{eV}$. Il semble difficile d'améliorer grandement la dispersion énergétique de ces cathodes.

Une autre solution pour produire des électrons consiste à utiliser l'émission de champ dont le principe est le suivant : lorsqu'un champ électrique élevé (de l'ordre de $10^{9} \mathrm{~V} \mathrm{~m}^{-1}$ ) est appliqué à l'extrémité d'une pointe, un courant électronique est émis à travers la barrière de potentiel, même à température «nulle» ([1], Fig. 1b). La densité de

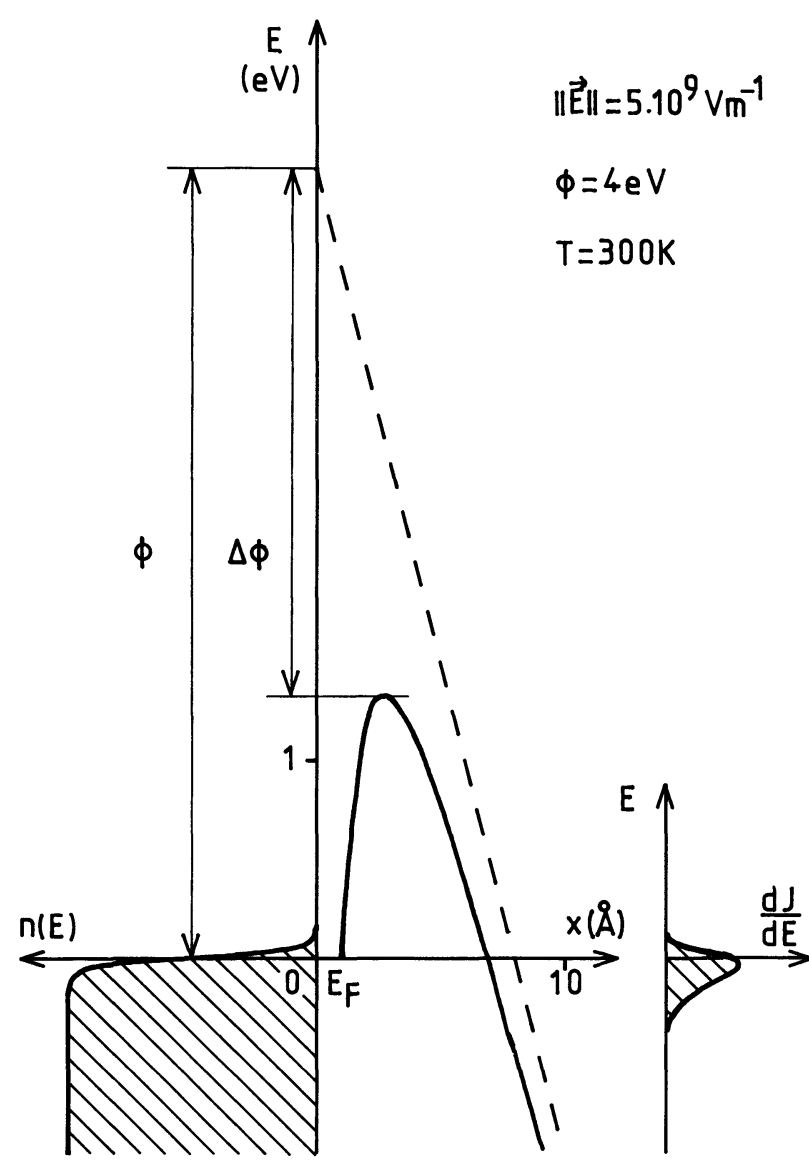

b)

[Energy diagram corresponding to thermoelectronic (1a) and field emission (1b).]

courant obéit à la loi de Fowler-Nordheim :

$$
J=k_{1} \frac{\|\mathbf{E}\|^{2}}{\phi} \exp \left(-k_{2} \frac{\phi^{3 / 2}}{\|\mathbf{E}\|}\right),
$$

où $\mathbf{E}$ est le champ électrique à l'extrémité de la pointe, $\phi$ le travail de sortie de celle-ci, $k_{1}$, $k_{2}$ deux fonctions de $\|\mathbf{E}\|$ et $\phi$ constantes au premier ordre. Dans les montages classiques, le champ est créé par une anode située à une distance faible (1 mm) en face d'une pointe de rayon de courbure $1000 \AA ̊$ environ. L'anode est percée d'une ouverture permettant l'extraction du faisceau. Quoique la densité de courant puisse être très forte (de l'ordre de $10^{8} \mathrm{~A} \mathrm{~m}^{-2}$ ), l'aire de la partie émissive de la pointe est très réduite et le courant extrait est donc faible : même pour des tensions d'anode élevées $(>1 \mathrm{kV})$, le courant est relativement faible (1 à $10 \mu \mathrm{A})$. L'avantage de ce dispositif réside dans la forte brillance de la source [2] et a conduit à une utilisation en microscopie électronique ainsi qu'en microlithographie. En ce qui concerne les applications aux faibles énergies $(\sim 10 \mathrm{eV})$, la bonne résolution en énergie (jusqu'à moins de $0,2 \mathrm{eV}$ ) et le fait que la source émette même à très basse température 
constituent des avantages. Cependant la tension d'extraction élevée est un inconvénient car la majeure partie du courant est perdue lors du retardement du faisceau.

Une solution permettant de surmonter ce handicap est apparue au cours de ces dernières années, grâce aux possibilités offertes par le développement de la microélectronique [3, 4]. Cette solution consiste à associer un grand nombre de pointes (par exemple 10000 sur une surface de $1 \mathrm{~mm}^{2}$ ), chacune de ces pointes (de rayon de courbure $<1000 \AA$ ) étant située face à une anode d'extraction placée au plus près (moins d'un micron) et appelée grille, comme on peut le voir sur la Fig. 2. Pour donner un ordre de grandeur, une tension d'extraction de $100 \mathrm{~V}$ permet d'obtenir, pour un ensemble de 10000 pointes, un courant de l'ordre du milliampère, correspondant à un courant moyen de 0,1 microampère par pointe. Ainsi la plus faible émission de chaque pointe, due à la plus faible tension d'extraction est compensée par le nombre de celles-ci. Le retardement des électrons de $100 \mathrm{eV}$ à $10 \mathrm{eV}$ et leur focalisation ne posent pas de problèmes insurmontables.

Jusqu'à présent ce type de cathodes a trouvé ses applications dans divers domaines allant des écrans plats [4] à la spectroscopie ionique [5]. Les difficultés demeurent dans les domaines où la dispersion en énergie est un paramètre critique [6].

Dans ce qui suit, les caractéristiques de ces cathodes à émission de champ, développées dans nos laboratoires (LETI), sont décrites, en reprenant certains concepts présentés dans la référence [3]; puis le problème de la mesure de la dispersion en énergie des électrons est abordé. Nos résultats seront comparés à ceux d'un autre travail [6], dans lequel les auteurs s'étaient attachés à mesurer la dispersion énergétique de ce type de cathodes en vue d'une application à la physique atomique.

\section{Caractéristiques des cathodes à effet de champ.}

\subsection{CARACTÉRISTIQUE DE L'ÉMISSION PAR EFFET} DE CHAMP. - Avant d'aborder les problèmes spécifiques des cathodes contenant un grand nombre de pointes, précisons les caractéristiques générales de l'émission de champ, telles qu'elles résultent des calculs standard présentés par Young [7] ou Gadzuk et Plummer [8] pour une géométrie plane.

A une température nulle, la densité de courant s'écrit :

$$
J_{0}=\frac{A\|\mathbf{E}\|^{2}}{\phi t^{2}(y)} \exp \left(-B \frac{\phi^{3 / 2}}{\|\mathbf{E}\|} v(y)\right) .
$$

Lorsque le travail de sortie est exprimé en électron-volts, les coefficients $A$ et $B$ valent :

$$
A=1,54 \times 10^{-6} ; \quad B=6,83 \times 10^{9} .
$$

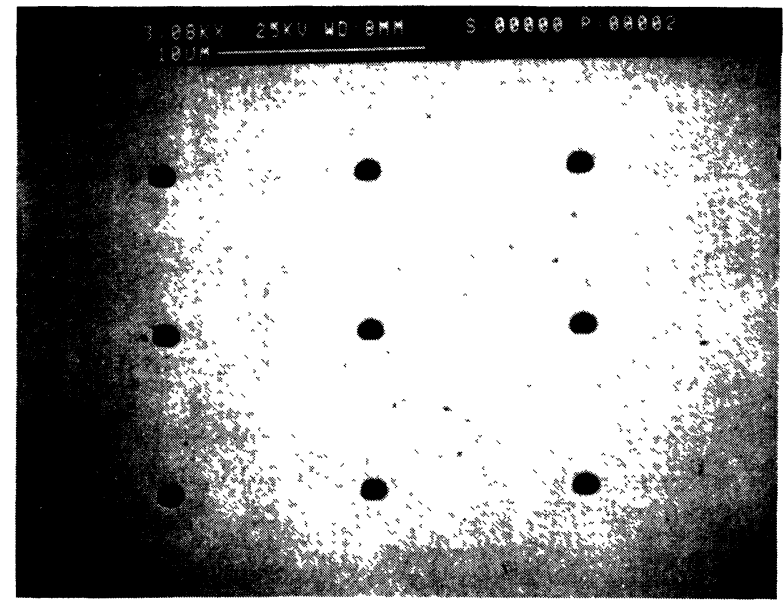

a) grossissement

magnification \#3000 x
pas
pitch $=10 \mu \mathrm{m}$

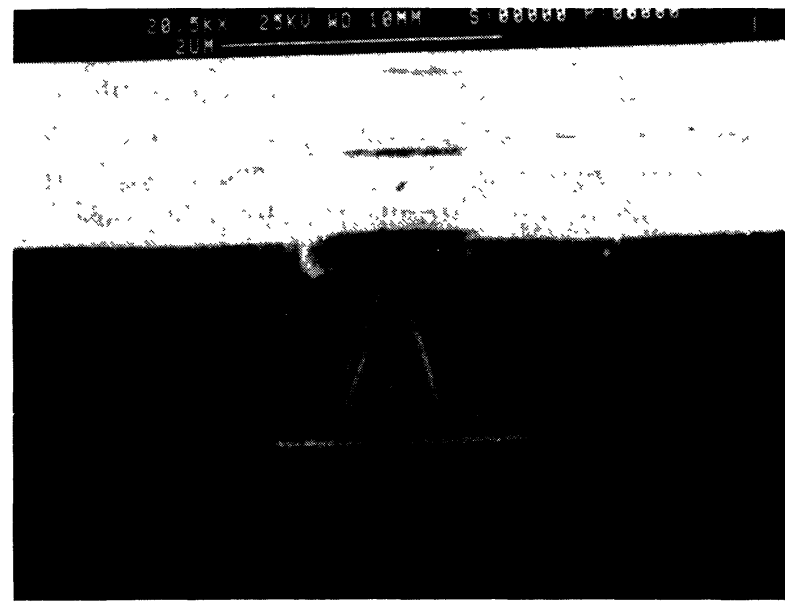

b) grossissement

$$
\begin{aligned}
& \text { magnification } \# 20000 \times \\
& \varnothing \text { grille } \\
& \varnothing \text { grid }=1.4 \mu \mathrm{m}
\end{aligned}
$$

Fig. 2. - Microphotographies de cathodes à émission de champ (microscopie électronique à balayage). Noter la parfaite régularité des pointes qui sont pratiquement toutes identiques à celle représentée en $2 b$ ).

[Microphotographs of field emission cathodes (scanning electron microscopy). Note the perfect regularity of the tips which look like the one represented in Fig. 2b.]

Les fonctions $t(y)$ et $v(y)$ permettent de tenir compte du potentiel de charge image et peuvent être approchées par les formules suivantes [3] :

$$
t^{2}(y)=1,1 \quad \text { et } \quad v(y)=0,95-y^{2},
$$

où $y$ est l'abaissement relatif de la barrière de 
potentiel induit par la charge image (effet Schottky).

$$
y=3,79 \times 10^{-5}\|\mathbf{E}\|^{1 / 2} / \phi_{(\mathrm{eV})} .
$$

En utilisant ces approximations on obtient donc :

$$
\begin{aligned}
J_{0}=1,4 \times 10^{-6} & \frac{\|\mathbf{E}\|^{2}}{\phi_{(\mathrm{eV})}} \exp \left(9,81 \phi_{(\mathrm{cV})}^{-1 / 2}\right) \times \\
& \times \exp \left(-6,49 \times 10^{9} \frac{\phi_{(\mathrm{eV})}^{3 / 2}}{\|\mathbf{E}\|}\right) .
\end{aligned}
$$

A température non nulle, il faut introduire un nouveau paramètre :

$$
d_{(\mathrm{cV})}=0,93 \times 10^{-10} \frac{\|\mathbf{E}\|}{\phi_{(\mathrm{eV})}^{1 / 2}} .
$$

La densité de courant émise à la température $T$ est alors, en exprimant $k T$ en électron-volts, et pourvu que $d$ soit plus grand que $k T[2]$ :

$$
J=J_{0} \frac{\pi k T / d}{\sin (\pi k T / d)} .
$$

Les caractéristiques expérimentales font intervenir le courant émis $I$ et la tension $V$ appliquée entre la pointe et l'anode d'extraction (appelée grille pour nos cathodes). Le courant est relié à la densité de courant par :

$$
I=J \cdot S,
$$

où $S$ est l'aire de la surface émissive. Pour cela on suppose que la densité de courant est constante sur toute cette surface, située à l'extrémité de la pointe. La tension appliquée entre la pointe et la grille est reliée au champ à l'extrémité de la pointe par :

$$
\|\mathbf{E}\|=\beta\left(V_{\mathrm{g}}-V_{\mathrm{p}}\right)=\beta V .
$$

Si maintenant l'on introduit les relations (2.8) et (2.9) dans l'expression de $J_{0}$, on trouve, pour une pointe ou pour un ensemble de pointes supposées identiques, la caractéristique $I(V)$ suivante :

$$
\begin{aligned}
I=1,4 \times 10^{-6} & \frac{\beta^{2} V^{2} S}{\phi} \exp \left(9,81 \phi^{-1 / 2}\right) \times \\
& \times \exp \left(-6,49 \times 10^{9} \frac{\phi^{3 / 2}}{\beta V}\right) .
\end{aligned}
$$

La représentation habituelle de cette caractéristique consiste à tracer $\log \left(I / V^{2}\right)$ en fonction de $1 / V$ :

$$
\begin{aligned}
\log \left(\frac{I}{V^{2}}\right)= & \\
=\log \left(1,4 \times 10^{-6}\right. & \left.\frac{\beta^{2} S}{\phi} \exp \left(9,81 \phi^{-1 / 2}\right)\right)- \\
& -2,82 \times 10^{9} \frac{\phi^{3 / 2}}{\beta V}, \quad(2.11)
\end{aligned}
$$

qui est une droite, dite de Fowler-Nordheim [1]. La courbe obtenue expérimentalement est en général effectivement une droite même lorsque $I$ varie sur plusieurs décades. La pente de la droite vaut :

$$
p=-2,82 \times 10^{9} \frac{\phi^{3 / 2}}{\beta}
$$

tandis que la valeur extrapolée en $1 / V=0$ est :

$$
\left(\frac{I}{V^{2}}\right)_{1 / V=0}=1,4 \times 10^{-6} \frac{\beta^{2} S}{\phi} \exp \left(9,81 \phi^{-1 / 2}\right) \text {. }
$$

Il est clair qu'à partir de ces deux seules données expérimentales il n'est pas possible de remonter aux trois paramètres $\beta, S$ et $\phi$.

Cependant on peut éliminer $\beta$ entre les deux relations précédentes [9] :

$$
\begin{aligned}
\left(\frac{I}{V^{2}}\right)_{1 / V=0} & \times p^{2}=1,11 \times \\
& \times 10{ }^{13} S \phi^{2} \exp \left(9,81 \phi^{-1 / 2}\right) .
\end{aligned}
$$

La fonction $\phi^{2} \exp \left(9,81 \phi^{-1 / 2}\right)$ vaut 2250 à $15 \%$ près lorsque $\phi$ varie de $3 \mathrm{eV}$ à plus de $10 \mathrm{eV}$ [3]. Comme le travail de sortie des pointes que nous utilisons est en général supérieur à $3 \mathrm{eV}$, cette condition est toujours vérifiée ; on peut alors en déduire la valeur de la surface d'émission :

$$
S=4 \times 10^{-17}\left(\frac{I}{V^{2}}\right)_{1 / V=0} \times p^{2} .
$$

En ce qui concerne le facteur de conversion $\beta$ et le travail de sortie $\phi$, on ne dispose que de la relation (2.12) qui les relie à la pente de la caractéristique.

2.2 Caractéristiques des CATHodes. - La caractéristique de Fowler Nordheim d'une cathode constituée de $10^{4}$ pointes en carbure de titane, déposées sur un substrat de silicium, est présentée comme exemple sur la figure 3 (cathode $n^{\circ} 1$, courbe en traits pleins). Si l'on prolonge la partie de la courbe comprise entre $70 \mathrm{~V}$ et $90 \mathrm{~V}$ la pente et l'intersection avec l'axe des ordonnées valent :

$$
\begin{aligned}
p & =-330, \\
\left(\frac{I}{V^{2}}\right)_{1 / V=0} & =5,3 \times 10^{-5} \mathrm{~A} \mathrm{~V}^{-2}, \text { d'où : } \\
S & =2,3 \times 10^{-16} \mathrm{~m}^{2},
\end{aligned}
$$

ce qui correspond, si on suppose que toutes les pointes sont identiques et émettent en permanence, à une surface émissive par pointe :

$$
s=2,3 \AA^{2},
$$

de l'ordre de la «surface d'un atome » (pour comparaison $s=12 \AA^{2}$ pour des pointes similaires [3]). 


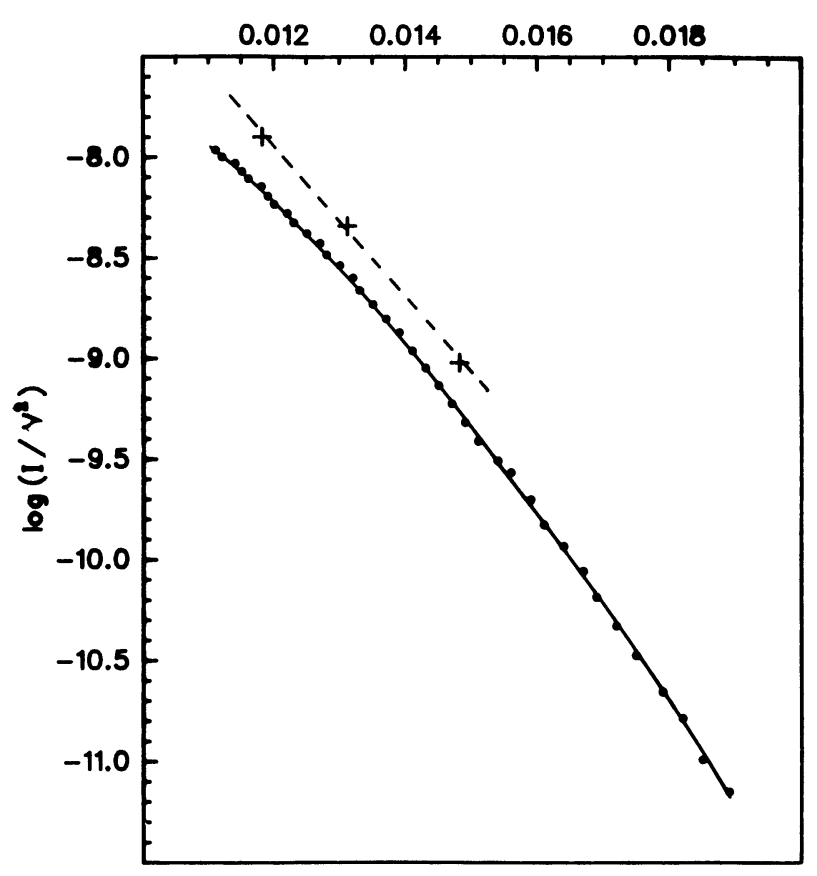

$1 / v\left(v^{-1}\right)$

Fig. 3. - Caractéristique de Fowler-Nordheim d'une cathode à émission de champ (cathode $\left.\mathrm{n}^{\circ} 1\right)$. (—) non corrigée ; (----) corrigée, en tenant compte de la chute de tension à l'interface.

[Fowler-Nordheim plot of a field emission cathode (cathode $\left.\mathrm{n}^{\circ} 1\right)$. ( $\longrightarrow$ ) uncorrected ; (-----) corrected for the interface voltage drop.]

A partir de $p$, et en supposant que le travail de sortie du carbure de titane est compris entre 4 et $5 \mathrm{eV}$ [10], le facteur $\beta$ vaut, d'après (2.12) :

$$
\beta=6,9 \times 10^{7} \mathrm{~m}^{-1} \text { à } 9,6 \times 10^{7} \mathrm{~m}^{-1}
$$

(à comparer à $6 \times 10^{7} \mathrm{~m}^{-1}[3]$ ).

Pour prendre en compte les incertitudes sur la détermination de $p$ et $I / V^{2}$ considérons maintenant la partie de la caractéristique comprise entre $50 \mathrm{~V}$ et $70 \mathrm{~V}$. On a alors :

$$
\begin{gathered}
p=-460,\left(\frac{I}{V^{2}}\right)_{1 / V=0}=3,4 \times 10^{-3} \mathrm{~A} \mathrm{~V}^{-2} \text {, d'où : } \\
S=2,9 \times 10^{-14} \mathrm{~m}^{2}, \text { soit } s=290 \AA^{2},
\end{gathered}
$$

et :

$$
\beta=4,9 \times 10^{7} \text { à } 6,8 \times 10^{7} \mathrm{~m}^{-1}, \quad \text { pour } \phi=4 \text { à } 5 \mathrm{eV}[10] .
$$

Les valeurs expérimentales obtenues à forte tension sont raisonnablement proches des résultats de [3]. Cet accord est attendu, si l'on considère que la technologie de fabrication et les dimensions géométriques du dispositif (voir Fig : 2b), ainsi que les champs électriques appliqués, sont très proches dans les deux cas.
Cependant ces résultats et ceux obtenus à basse tension sont en désaccord avec les valeurs calculées : Spindt et al. [3] ont calculé numériquement le champ électrique en tout point de la surface de la pointe et en ont déduit la surface d'émission par pointe ainsi que le facteur $\beta$ :

$$
s=6,3 \times 10^{-15} \mathrm{~m}^{2} \text { et } \beta=1,25 \times 10^{7} \mathrm{~m}^{-1},
$$

ce qui donnerait :

$$
\left(\frac{I}{V^{2}}\right)_{1 / V=0}=4,6 \times 10^{-5} \mathrm{~A} \mathrm{~V}^{-2},
$$

et

$$
p=-1800, \text { pour } \phi=4 \mathrm{eV} .
$$

Les valeurs expérimentales sont donc, compte tenu des incertitudes, très éloignées des valeurs calculées : on a un désaccord de trois à quatre ordres de grandeurs sur $s$, et d'un facteur 4 à 8 sur $\beta$, se traduisant principalement par une pente anormalement faible. Pour concilier les valeurs expérimentales de la pente et la valeur théorique de $\beta$, il faudrait supposer (Eq. (2.12)) que le travail de sortie du matériau est $\phi=1,3$ à $1,6 \mathrm{eV}$, ce qui est improbable, même en tenant compte d'une modification de la valeur du travail de sortie par suite de la contamination de la pointe par les gaz résiduels habituellement présents dans un vide de $10^{-9}$ mbar (notons d'ailleurs que ces résultats ne sont pas modifiés même lorsque le vide est de l'ordre de $1 \times 10^{-10}$ mbar).

L'explication de ces écarts importants est malaisée, du fait, entre autres, de la présence du grand nombre de pointes que l'on ne peut pour l'instant tester à la fois individuellement et en groupe. On peut cependant avancer quelques hypothèses.

1) Pourcentage de pointes défectueuses. - Lors de la fabrication des pointes, et lors des premières heures de fonctionnement, un certain nombre de pointes ne sont pas correctement formées, ou sont détruites, ce qui est vérifié en observant les cathodes au microscope électronique à balayage avant et après fonctionnement. La fraction de pointes défectueuses est de l'ordre de quelques pour cent mais ne dépasse pas $10 \%$. Cela peut en partie expliquer les faibles surfaces d'émission observées, mais ne peut changer l'ordre de grandeur des résultats.

2) Effet statistique sur les paramètres d'émission. La caractéristique expérimentale $\log I / V^{2}-1 / V$ d'une cathode comprenant un grand nombre de pointes est une droite. Cela ne signifie pas que les pointes soient semblables. En effet Tomaschke et Alpert [11] ont montré, que, même pour un ensemble de pointes avec des surfaces émissives et des facteurs $\beta$ très différents, la caractéristique est presque une droite, correspondant à un facteur $\beta$ apparent proche de la valeur maximale de $\beta$, et à 
une surface d'émission apparente inférieure à la surface totale d'émission. Cela est dû au fait que le courant émis varie très fortement avec le facteur $\beta$, pour une tension donnée, et qu'ainsi n'interviennent que les pointes de $\beta$ élevé. Cela expliquerait à la fois la faible surface d'émission et la valeur élevée du facteur $\beta$ apparent. On peut soulever cependant une objection : pour une cathode donnée les dimensions des pointes réalisées ici sont peu dispersées par rapport aux dimensions des projections étudiées dans [11], du fait de la technologie de réalisation; par conséquent l'influence de ce phénomène est certainement bien plus faible que pour le cas cité dans [11]. En particulier un facteur $\beta$ quatre fois plus élevé que la valeur calculée ne peut être attribué à une dispersion des dimensions.

3) Augmentation locale de l'émission. - Les auteurs de la référence [3] suggèrent que l'émission a lieu sur quelques sites atomiques situés à l'extrémité des pointes. En effet, la présence de microstructures à la surface de la pointe entraînerait une augmentation locale du champ, d'où un facteur $\beta$ élevé. Dans ces conditions il suffirait de quelques sites atomiques par pointe en moyenne (moyenne statistique sur l'ensemble des pointes, et moyenne temporelle) pour obtenir le courant observé. Le processus de formation de ces microstructures peut être relié à l'échauffement de la pointe résultant de la forte valeur du champ (voir plus loin). Leur destruction aurait lieu lorsque le champ dépasse la valeur de claquage.

4) Influence des atomes adsorbés. - Le coefficient de transmission de la barrière de potentiel que franchissent les électrons est profondément modifié par l'adsorption d'atomes et de molécules à la surface de la pointe : la densité de courant émis peut alors être augmentée ou diminuée sans que cela soit attribuable à une variation du travail de sortie [12]. Si les atomes adsorbés sont « légers » la pente de la caractéristique de Fowler-Nordheim est peu affectée ; dans le cas de l'adsorption d'atomes métalliques, la pente peut être abaissée d'un facteur 2 [12].

En l'état actuel des choses et bien que des observations aient été faites sur l'émission (et les fluctuations d'émissions) en fonction de la température et des conditions de vide pour une micropointe il ne nous est pas possible de privilégier une hypothèse plutôt qu'une autre.

Un dernier phénomène spécifique aux dispositifs où les pointes sont évaporées sur un substrat peut induire des erreurs sur les caractéristiques d'émission : c'est la résistance d'interface entre le substrat et la pointe. Ce problème sera abordé plus en détail dans le paragraphe concernant la dispersion en énergie. En ce qui concerne son influence sur la caractéristique $I(V)$, il est clair qu'une chute de tension entre le substrat et la pointe diminue la tension grille-pointe effective. Comme cette chute de tension est plus forte à haute tension (et fort courant), la caractéristique $I(V)$ réelle a une pente plus forte et une intersection avec l'axe des ordonnées plus haute que la caractéristique mesurée. A travail de sortie donné, le facteur de conversion $\beta$ est surévalué, et la surface d'émission sous-évaluée si l'on ne tient pas compte de cette chute de tension. Pour la cathode considérée (cathode $n^{\circ} 1$ ), la chute de potentiel a été mesurée par la méthode de retardement (voir plus loin) et on a trouvé $\Delta V=$ $2,5 \mathrm{~V} ; 3,8 \mathrm{~V}$ et $5,5 \mathrm{~V}$ pour $V=70 \mathrm{~V} ; 80 \mathrm{~V}$ et $90 \mathrm{~V}$, ce qui donne une caractéristique corrigée représentée en traits interrompus sur la figure 3. Cette correction permet d'évaluer à nouveau la pente $p$ et le facteur $\left(I / V^{2}\right)_{1 / V=0}$ :

$$
\begin{aligned}
p & =-387 \text { au lieu de }-330, \\
\left(\frac{I}{V^{2}}\right)_{1 / V=0}=5,2 \times 10^{-4} & \text { au lieu de } 5,3 \times 10^{-5} \mathrm{~A} \mathrm{~V}^{-2},
\end{aligned}
$$

de sorte que le facteur $\beta$ et la surface d'émission deviennent :

$$
\begin{aligned}
& \beta=5,8 \times 10^{7} \mathrm{~m}^{-1}, \text { au lieu de } 6,9 \times 10^{7} \mathrm{~m}^{-1}, \\
& S=31 \times 10^{-16} \mathrm{~m}^{2}, \text { au lieu de } 2,6 \times 10^{-16} \mathrm{~m}^{2},
\end{aligned}
$$

soit $30 \AA^{2}$ au lieu de $2,5 \AA^{2}$ par pointe.

En conclusion, il paraît délicat de séparer l'influence des divers facteurs d'erreur sur l'évaluation de la surface d'émission par pointe ainsi que sur le facteur $\beta$. Les résultats présentés ici sont à considérer comme une première évaluation destinée à exposer la méthode de calcul et les problèmes rencontrés. Une fois le problème des chutes de tension résolu par l'utilisation de substrats métalliques très conducteurs, il sera souhaitable de reprendre l'étude des caractéristiques de Fowler-Nordheim et l'évaluation de la surface d'émission.

\section{Energie et dispersion énergétique des électrons.}

Les méthodes de mesure de la dispersion en énergie d'un faisceau d'électrons que nous avons utilisées peuvent être classées en trois catégories :

1) l'analyse directe par la méthode du potentiel retardateur,

2) l'analyse directe par un spectromètre à déflection,

3) l'analyse indirecte fondée sur un processus physique connu, ici la photoémission inverse.

Avant de présenter les résultats obtenus par ces trois méthodes, voyons les valeurs théoriques qu'on peut attendre pour la dispersion en énergie des électrons émis par émission de champ.

3.1 DiSPERSION EN ÉNERGIE THÉORIQUE. - La distribution en énergie théorique des électrons émis 
par émission champ est donné $[7,13]$ par la relation suivante :

$$
\frac{\mathrm{d} J}{\mathrm{~d} E}=\frac{J_{0} \exp (\varepsilon / d)}{d[1+\exp (\varepsilon / k T)]},
$$

où $J_{0}$ est la densité de courant totale à $T=$ $0 \mathrm{~K}$, donnée par (2.1), $d$ le paramètre défini par la relation (2.6), $T$ la température absolue et :

$$
\varepsilon=E-E_{\mathrm{F}},
$$

l'énergie relative des électrons par rapport au niveau de Fermi de la pointe (voir Fig. 4).

A température nulle la valeur de la distribution en énergie est nulle au-dessus du niveau de Fermi, maximale au niveau de Fermi, et décroît exponentiellement au-dessous. La largeur totale à mi-hauteur vaut :

$$
\Delta E_{0}=d \ln 2 \simeq 0,64 \times 10^{-10} \frac{\|\mathbf{E}\|}{\phi_{(\mathrm{eV})}^{1 / 2}} .
$$

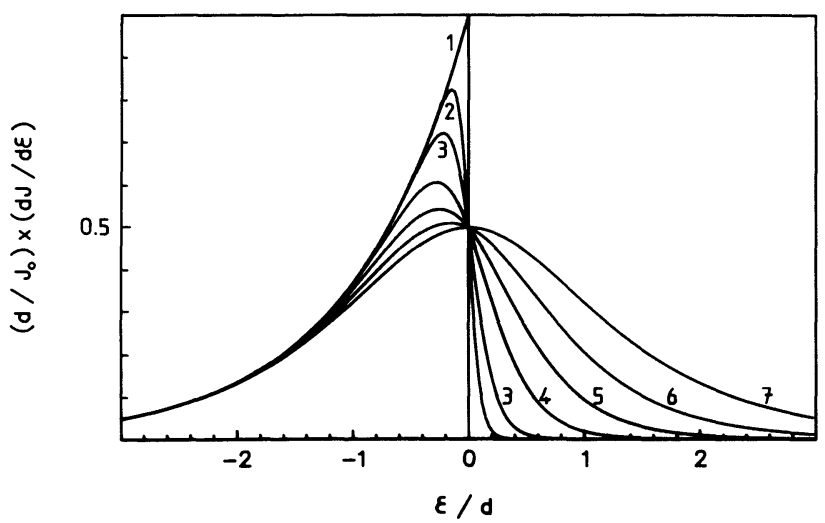

Fig. 4. - Distribution théorique de l'énergie totale des électrons émis par émission de champ. 1) $k T=0$;2) $k T=$ $0,048 d$; 3) $k T=0,1 d$; 4) $k T=0,2 d$; 5) $k T=0,3 d$; 6) $k T=0,4 d$; 7) $k T=0,5 d$ (d'après Swanson et Crouser [14]).

[Theoretical total-energy-distribution of field emitted electrons. 1) $k T=0$; 2) $k T=0,048 d$; 3) $k T=0,1 d$;4) $k T=0,2 d$; 5) $k T=0,3 d$; 6) $k T=0,4 d$; 7) $k T=0,5 d$ (after Swanson and Crouser [14]).]

Lorsque la température augmente, la distribution en énergie des électrons émis s'élargit [14] comme on peut le voir sur la figure 4 où est représentée, pour une valeur donnée du paramètre $d$, l'évolution de la distribution en énergie en fonction de la température.

En outre, l'énergie moyenne par rapport au niveau de Fermi des électrons émis augmente avec la température suivant la relation [15] :

$$
\bar{\varepsilon}=-\pi k T \operatorname{cotg} \frac{\pi k T}{d}
$$

Lorsque la température est basse $(2 k T<d)$, $\bar{\varepsilon}$ est négative, et l'émission de champ tend à échauffer la pointe. Lorsque la température est élevée $(2 k T>d), \bar{\varepsilon}$ est positive et l'émission de champ tend à la refroidir (en moyenne, les électrons «emportent» une quantité d'énergie positive). Si l'on suppose, pour simplifier, que la pointe est isolée thermiquement, et que sa température initiale est $T=0 \mathrm{~K}$, l'émission de champ va tout d'abord se traduire par un échauffement, jusqu'à un équilibre thermodynamique, où l'énergie « emportée » par les électrons émis au-dessus du niveau de Fermi compense l'énergie reçue par la pointe du fait de l'émission d'électrons au-dessous du niveau de Fermi. Si au contraire la pointe est initialement à une température élevée $(2 k T>d)$ l'émission de champ la refroidit jusqu'à l'équilibre. Ce phénomène porte le nom d'effet Nottingham, et la température d'équilibre est appelée température d'inversion ou température critique $T_{\mathrm{c}}$. Pour une pointe isolée la condition d'équilibre est $\bar{\varepsilon}=0$, ce qui apparaît clairement sur la figure 4 , et l'on a :

$$
d=2 k T_{\mathrm{c}}, \quad \text { soit } \quad T_{\mathrm{c}}=5,32 \times 10^{-7} \frac{\|\mathbf{E}\|}{\phi_{(\mathrm{eV})}^{1 / 2}},
$$

(En toute rigueur il faudrait tenir compte de la dissipation d'énergie par effet Joule dans la pointe, et de la conduction thermique de la chaleur vers le substrat [15, 16].)

Quant à la largeur à mi-hauteur de la distribution en énergie, on $\mathrm{a}$, pour $T=T_{\mathrm{c}}$

$\Delta E_{\mathrm{c}}=5,27 k T_{\mathrm{c}}=2,63 \mathrm{~d}=2,44 \times 10^{-10} \frac{\|\mathbf{E}\|}{\phi_{(\mathrm{eV})}^{1 / 2}}$.

Ainsi peut-on connaître immédiatement la valeur de la dispersion en énergie pour $T=0 K$ (Eq. (3.2)) et pour $T=T_{\mathrm{c}}$ (Eq. (3.5)). On constate [17] que, pour un champ donné, tant que la température de la cathode est inférieure à $T_{\mathrm{c}}(k T<d / 2)$, la dispersion en énergie augmente de manière monotone avec la température entre $T=0 \mathrm{~K}$ et $T=T_{\mathrm{c}}$. Par conséquent les formules 3.2 et 3.5 donnent les limites inférieures et supérieures de la dispersion énergétique (il pourrait arriver que la condition $k T<$ $d / 2$ ne soit pas remplie, si par exemple on chauffait la pointe).

Considérons, comme exemple, les données (corrigées) concernant la cathode étudiée au paragraphe précédent (cathode $\mathrm{n}^{\circ} 1$ ). On peut évaluer le champ électrique $\left(\|\mathbf{E}\|=\beta V ; \beta=5,8 \times 10^{7} \mathrm{~m}^{-1}\right)$, la température d'inversion $T_{\mathrm{c}}$ (Eq. (3.4), avec $\phi=4 \mathrm{eV}$ ), la dispersion en énergie $\Delta E_{0}$ à $T=0 \mathrm{~K}$ et $\Delta E_{\mathrm{c}}$ à $T=T_{\mathrm{c}}$. Ces résultats sont présentés sur le tableau $\mathrm{I}$.

En ce qui concerne le maximum de la distribution en énergie $\varepsilon_{\mathrm{m}}$, un calcul simple montre qu'il est compris, pour $d$ fixé et $T<T_{\mathrm{c}}$, entre 0 (pour 
Tableau I. - Dispersion énergétique calculée à $T=$ $0 \mathrm{~K}$ et à $T=T_{\mathrm{c}}$, cathode $\mathrm{n}^{\circ} 1$.

[Energetic spread, calculated for $T=0 \mathrm{~K}$ and $T=T_{\mathrm{c}}$, cathode $\left.\mathrm{n}^{\circ} 1\right]$.

\begin{tabular}{|c|c|c|c|r|c|}
\hline$V(\mathrm{~V})$ & $E\left(\mathrm{~V} \mathrm{~m}^{-1}\right)$ & $d(\mathrm{eV})$ & $\Delta E_{0}(\mathrm{eV})$ & $T_{\mathrm{c}}(\mathrm{K})$ & $\Delta E_{\mathrm{c}}(\mathrm{eV})$ \\
\hline 30 & $1,7 \times 10^{9}$ & 0,08 & 0,06 & 460 & 0,21 \\
70 & $4,1 \times 10^{9}$ & 0,19 & 0,13 & 1080 & 0,49 \\
90 & $5,2 \times 10^{9}$ & 0,24 & 0,17 & 1390 & 0,63 \\
\hline
\end{tabular}

$T=0$ ou $T=T_{\mathrm{c}}$ ) et $0,28 d$ environ (pour $T \simeq$ $0,44 T_{\mathrm{c}}$, voir courbe 2, Fig. 4). Dans l'exemple cité (cathode 1), l'écart du maximum par rapport au niveau de Fermi reste toujours inférieur à $0,07 \mathrm{eV}$. Cet écart est très faible par rapport aux décalages provenant de la chute de potentiel à l'interface comme on le verra plus loin. On peut donc considérer que, dans le modèle de Fowler-Nordheim, les électrons sont émis au niveau de Fermi tant que $T<T_{\mathrm{c}}$ (et ceci bien sûr compte tenu de la résolution des appareils dont nous disposons).

Enfin, il faut noter que dans le cas d'une adsorption d'atomes à la surface de la pointe, le modèle présenté ci-dessus n'est pas complet, et qu'il peut apparaître à la fois une modification dans l'allure de la dispersion énergétique et un déplacement supplémentaire de son maximum [12]. Pour déceler la modification de la distribution il faudrait étudier une pointe unique à l'aide d'un spectromètre conçu spécialement (voir revue dans [8]). Quant au décalage, qui est principalement observé lors de l'adsorption d'atomes métalliques (ce qui n'est probablement pas le cas ici) il est de l'ordre de $0,5 \mathrm{eV}$ au maximum $[8,12]$; comme on le verra ci-dessous, cela reste encore faible par rapport à l'effet de la chute de potentiel à l'interface.

La principale conclusion de ce paragraphe est qu'il est relativement simple de calculer une fourchette de valeurs pour la dispersion en énergie des électrons émis, tandis que le calcul exact de celle-ci suppose un bilan thermique précis des pointes (qui pourrait s'inspirer de la référence [15]) ainsi que la prise en compte des phénomènes d'adsorption.

\subsection{Mesure de la distribution EN ÉNERGIE.}

3.2.1 Méthode du potentiel retardateur. - Soit le montage expérimental de la figure 5a, dans lequel une anode est placée en face d'une cathode (constituée d'un grand nombre de pointes, une seule d'entre elles étant représentée). Soit $E_{\mathrm{Fp}}$ le niveau de Fermi des pointes, $E_{\mathrm{Fg}}$ celui de la grille servant d'anode d'extraction, et $E_{\mathrm{Fa}}$ celui de l'anode. On peut écrire les potentiels $V_{\mathrm{a}}-V_{\mathrm{p}}$ et $V_{\mathrm{g}}-V_{\mathrm{p}}$, auxquels sont portées l'anode et la grille par rapport aux pointes, de la manière suivante :

$$
\begin{aligned}
& E_{\mathrm{Fp}}-E_{\mathrm{Fa}}=e\left(V_{\mathrm{a}}-V_{\mathrm{p}}\right), \\
& E_{\mathrm{Fp}}-E_{\mathrm{Fg}}=e\left(V_{\mathrm{g}}-V_{\mathrm{p}}\right) .
\end{aligned}
$$

L'énergie cinétique des électrons à la sortie du dispositif d'extraction (disons à quelques microns en avant de la grille) est $e\left(V_{\mathrm{g}}-V_{\mathrm{p}}\right)-\phi_{\mathrm{g}}$ (Fig. 5b). Lorsque l'anode est portée à un potentiel supérieur à celui de la grille, comme c'est le cas sur la figure 5, les électrons émis par la pointe sont accélérés vers l'anode.

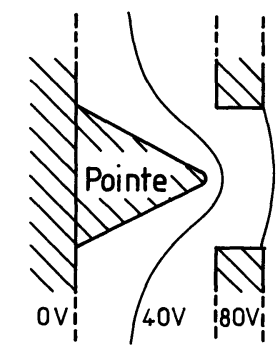

Substrat Grille
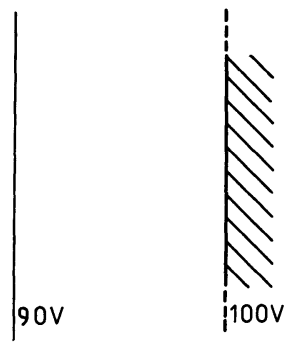

Anode

a)

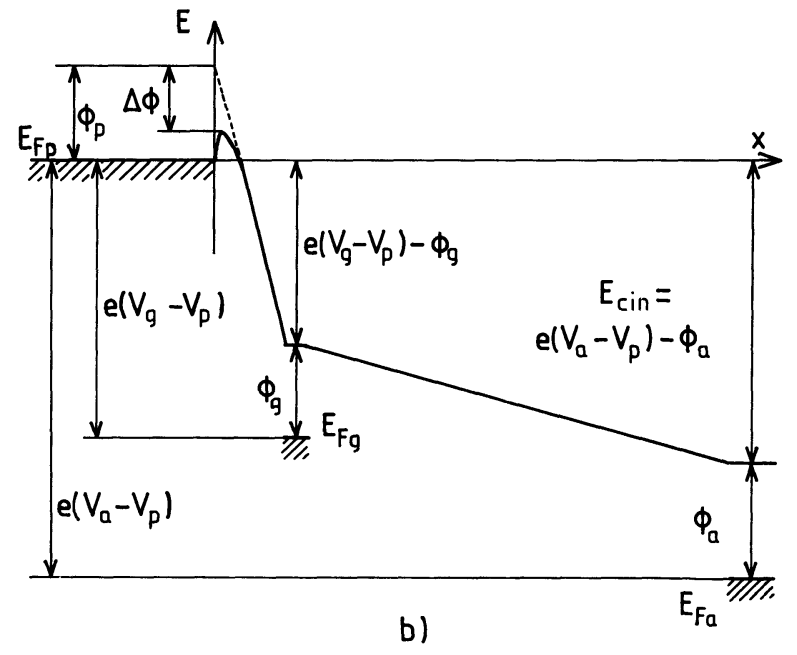

Fig. 5. - Forme des équipotentielles (5a) et diagramme d'énergie (5b) pour des électrons émis par émission de champ et accélérés vers une anode.

[Equipotential surfaces (5a) and energy diagram (5b) for field emitted electrons accelerated towards an anode.]

Supposons maintenant que la tension appliquée sur l'anode diminue et devienne inférieure à celle qui est appliquée sur la grille. Les électrons sont alors retardés, et ceux qui sont émis avec un angle « trop » fort par rapport à la normale aux anodes, ne peuvent atteindre l'anode collectrice, et retournent sur la grille. Ainsi, lorsque l'on abaisse la tension $V_{\mathrm{a}}-V_{\mathrm{p}}$, le courant d'anode $I_{\mathrm{a}}$ diminue-t-il. Lorsque $V_{\mathrm{a}}-V_{\mathrm{p}}$ devient «trop » faible, c'est-à-dire plus faible que le travail de sortie $\phi_{\mathrm{a}}$ de l'anode, ce courant devient nul (Fig. 6a). Le diagramme d'énergie correspondant au cas limite $e\left(V_{\mathrm{a}}-V_{\mathrm{p}}\right)=\phi_{\mathrm{a}}$ est 

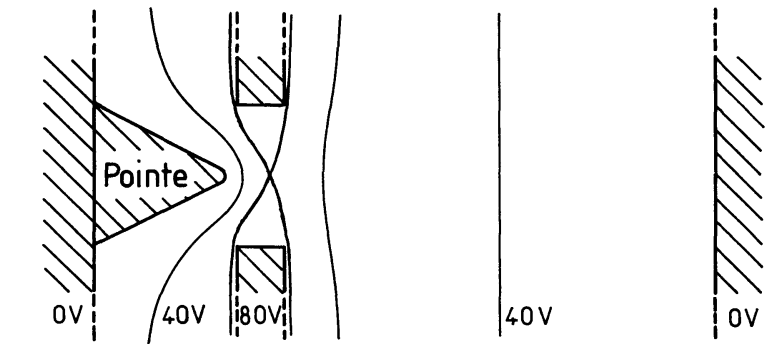

Substrat

Grille

a)

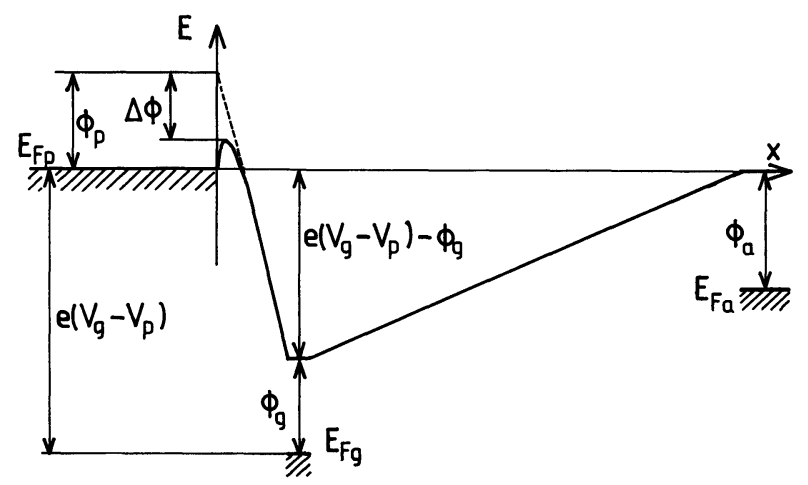

b)

Fig. 6. - Forme des équipotentielles (6a) et diagramme d'énergie (6b) correspondant à la condition de coupure dans une expérience de retardement.

[Equipotential surface (6a) and energy diagram (6b) corresponding to the cut-off condition for a retardingpotential experiment.]

représenté sur la figure $6 \mathrm{~b}$. Si l'anode est située suffisamment loin de la cathode, on peut montrer que la coupure est franche, et on peut alors mesurer la dispersion des électrons émis avec une précision de l'ordre de $1 \mathrm{eV}$. Pour obtenir une meilleure résolution, il faudrait rendre le faisceau parallèle à l'aide d'une optique appropriée $[13,14]$, la méthode simple présentée ici ne pouvant fournir des renseignements précis. Pour la cathode dont la caractéristique $I_{\mathrm{a}}\left(V_{\mathrm{g}}-V_{\mathrm{p}}\right)$ a été présentée au paragraphe 2 (cathode $\mathrm{n}^{\circ} 1$, Fig. 3), les courbes $I_{\mathrm{a}}\left(V_{\mathrm{a}}-V_{\mathrm{p}}\right)$ ont été tracées pour $V_{\mathrm{g}}-V_{\mathrm{p}}=70 \mathrm{~V}, 80 \mathrm{~V}$ et $90 \mathrm{~V}$ sur la figure 7 (l'anode utilisée est en or avec $\phi \simeq 5 \mathrm{eV}$ ). Plus exactement, c'est le rapport du courant $I_{\mathrm{a}}\left(V_{\mathrm{a}}-V_{\mathrm{p}}\right)$ au courant maximum extrait $I_{\mathrm{a} \max }$ (mesuré pour $V_{\mathrm{a}}-V_{\mathrm{p}} \simeq 200 \mathrm{~V}$ ), qui est tracé. Comme on le voit la coupure n'est pas très franche, ce qui empêche une mesure précise de la dispersion en énergie. Cela est dû au fait que les électrons sont émis dans un cône large (dont le demi-angle est de l'ordre de 30 degrés) de sorte que l'énergie cinétique normale $E_{x}=P_{x}^{2} / 2 \mathrm{~m}$ est très dispersée (même si l'énergie cinétique totale est constante), et que donc, lorsque $V_{\mathrm{a}}-V_{\mathrm{p}}$, diminue, le courant $I_{\mathrm{a}}$ décroît constamment. De plus on constate que la valeur de la tension d'anode $V_{\mathrm{a} 0}-V_{\mathrm{p}}$, au-dessous

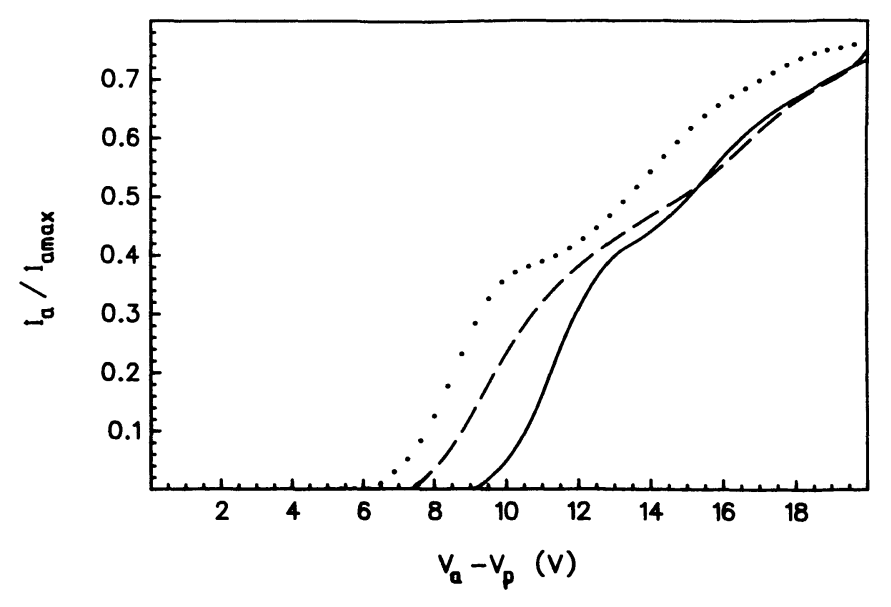

Fig. 7. - Rapport de courant $I_{\mathrm{a}} / I_{\mathrm{a} \max }$ en fonction de la tension de l'anode retardatrice $V_{\mathrm{a}}-V_{\mathrm{p}}$, pour différentes tensions de grille $V_{\mathrm{g}}-V_{\mathrm{p}}$. $(\ldots ..) V_{\mathrm{g}}-V_{\mathrm{p}}=70 \mathrm{~V} ;(----)$ $V_{\mathrm{g}}-V_{\mathrm{p}}=80 \mathrm{~V} ;\left(\stackrel{\mathrm{g}}{\longrightarrow} V_{\mathrm{g}}-V_{\mathrm{p}}=90 \mathrm{~V}\right.$.

[Current ratio $I_{\mathrm{a}} / I_{\mathrm{a} \max }$ as a function of the retarding anode voltage $V_{\mathrm{a}}-V_{\mathrm{p}}$, for various grid voltages $V_{\mathrm{g}}-V_{\mathrm{p}}$. (....) $\quad V_{\mathrm{g}}-V_{\mathrm{p}}=70 \mathrm{~V} ; \quad(---) \quad V_{\mathrm{g}}-V_{\mathrm{p}}=$ $\left.80 \mathrm{~V} ;(-) V_{\mathrm{g}}-V_{\mathrm{p}}=90 \mathrm{~V}.\right]$

de laquelle le courant $I_{\mathrm{a}}$ est nul (disons moins de $10 \%$ du courant maximum extrait $I_{\text {a max }}$ ), varie avec $V_{\mathrm{g}}-V_{\mathrm{p}}$, alors qu'on s'attend à avoir $e\left(V_{\mathrm{a} 0}-V_{\mathrm{p}}\right)=\phi_{\mathrm{a}}$ pour toute valeur de $V_{\mathrm{g}}-V_{\mathrm{p}}$. Dans le cas présent, $e\left(V_{\mathrm{a} 0}-V_{\mathrm{p}}\right)-\phi_{\mathrm{a}}$ vaut respectivement $2,5 \mathrm{eV}, \quad 3,8 \mathrm{eV}$ et $5,5 \mathrm{eV}$ pour $V_{\mathrm{g}}-V_{\mathrm{p}}=70 \mathrm{~V}, 80 \mathrm{~V}$ et $90 \mathrm{~V}$. Pour certaines séries de cathodes (substrats différents, paramètres géométriques modifiés...) cette différence peut atteindre $25 \mathrm{eV}$ pour $V_{\mathrm{g}}-V_{\mathrm{p}}=90 \mathrm{~V}$.

Ce phénomène est attribué à la nature de l'interface existant entre le substrat et les pointes. En effet, lorsque le substrat est en silicium (comme c'est le cas pour la cathode étudiée) une couche de silice peut se former sur celui-ci avant la fabrication de la pointe, ce qui entraîne l'existence d'une structure semiconducteur/isolant/métal à l'interface. On peut modéliser au premier ordre l'influence de cette interface par une résistance (éventuellement variable avec le courant), en sorte que la différence entre les niveaux de Fermi du substrat $\left(E_{\mathrm{Fs}}\right)$ et de la pointe $\left(E_{\mathrm{Fp}}\right)$ s'écrit :

$$
E_{\mathrm{Fs}}-E_{\mathrm{Fp}}=e R I_{\mathrm{p}}
$$

où $I_{\mathrm{p}}$ est le courant extrait, pour une tension de grille donnée, et $R$ la résistance d'interface. Il faut modifier les équations (3.6) et (3.7) :

$$
\begin{aligned}
& E_{\mathrm{Fs}}-E_{\mathrm{Fg}}=e\left(V_{\mathrm{g}}-V_{\mathrm{s}}\right), \\
& E_{\mathrm{Fs}}-E_{\mathrm{Fa}}=e\left(V_{\mathrm{a}}-V_{\mathrm{s}}\right),
\end{aligned}
$$

où $V_{\mathrm{g}}-V_{\mathrm{s}}$ et $V_{\mathrm{a}}-V_{\mathrm{s}}$ sont les tensions appliquées à la grille et à l'anode par rapport au substrat. D'autre part, l'énergie potentielle d'un électron dans le vide 
au voisinage de l'anode est $E_{\mathrm{va}}$, vérifiant :

$$
E_{\mathrm{va}}-E_{\mathrm{Fa}}=\phi_{\mathrm{a}},
$$

et la condition pour que les électrons, émis au niveau de Fermi de la pointe, puissent pénétrer dans l'anode est :

$$
E_{\mathrm{Fp}}-E_{\mathrm{Fa}}>\phi_{\mathrm{a}} .
$$

Le diagramme d'énergie résumant cette situation limite n'est plus celui de la figure $6 \mathrm{~b}$ mais celui de la figure 8 .

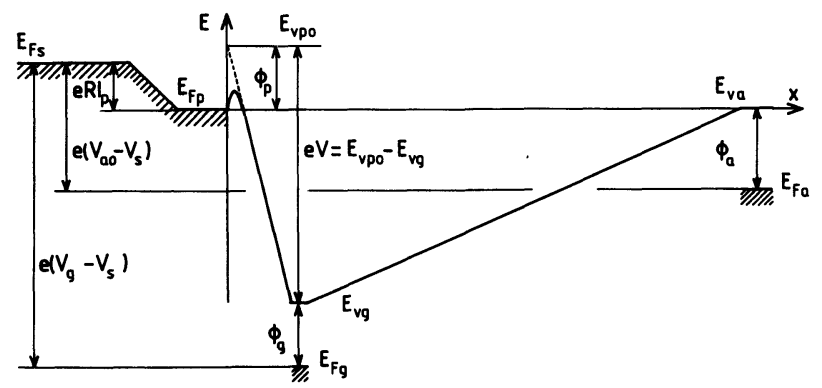

Fig. 8. - Diagramme d'énergie correspondant à la condition de coupure dans une expérience de retardement, dans le cas d'une chute de potentiel à l'interface substratpointe.

[Energy diagram corresponding to cut-off conditions for a retarding-potential experiment, in the case of a voltage drop at the substrate-tip interface.]

La tension de coupure $V_{\mathrm{a} 0}-V_{\mathrm{p}}$ correspondant à la condition (3.12) peut être obtenue à partir de (3.10) par :

$$
e\left(V_{\mathrm{a} 0}-V_{\mathrm{p}}\right)=E_{\mathrm{Fs}}-E_{\mathrm{Fp}}+E_{\mathrm{Fp}}-E_{\mathrm{Fa}},
$$

avec $E_{\mathrm{Fp}}=E_{\mathrm{va}}$, soit :

$$
e\left(V_{\mathrm{a} 0}-V_{\mathrm{p}}\right)=e R I_{\mathrm{p}}+\phi_{\mathrm{a}},
$$

Dans le cas considéré ici (cathode $\mathrm{n}^{\circ} 1$ ), où $e\left(V_{\mathrm{a} 0}-V_{\mathrm{p}}\right)-\phi_{\mathrm{a}}$ vaut respectivement $2,5 \mathrm{eV}$, $3,8 \mathrm{eV}$ et $5,5 \mathrm{eV}$ pour $E_{\mathrm{Fs}}-E_{\mathrm{Fg}}=e\left(V_{\mathrm{g}}-V_{\mathrm{s}}\right)=$ $70 \mathrm{eV}, 80 \mathrm{eV}$ et $90 \mathrm{eV}$ et $I_{\mathrm{p}}=6 \mu \mathrm{A}, 30 \mu \mathrm{A}, 90 \mu \mathrm{A}$, on en déduit la valeur de la résistance d'interface en fonction du courant : elle vaut $R=420 \mathrm{k} \Omega, 130 \mathrm{k} \Omega$ et $61 \mathrm{k} \Omega$ pour respectivement $I_{\mathrm{p}}=6 \mu \mathrm{A}, 30 \mu \mathrm{A}$ et $90 \mu \mathrm{A}$. En supposant que la résistance d'interface soit la même pour chaque pointe, on trouve une résistance de l'ordre de $10^{9} \Omega$ par pointe $\left(=10^{4} \times 100 \mathrm{k} \Omega\right)$, ce qui paraît énorme à première vue. Pourtant il suffit par exemple d'une couche isolante de résistivité $\rho=10^{6} \Omega \mathrm{m}$ et d'épaisseur $10 \AA$, à la surface de base de la pointe pour expliquer cette résistance.

Il apparaît de plus qu'une correction doit être effectuée sur la caractéristique de Fowler-Nordheim de la cathode. Pour une tension $V_{\mathrm{g}}-V_{\mathrm{s}}$ appliquée à la grille par rapport au substrat, la différence de potentiel $V$ qui impose la valeur du champ à l'extrémité de la pointe est donnée par (voir Fig. 8) :

$$
e V=E_{\mathrm{vp} 0}-E_{\mathrm{vg}},
$$

où $E_{\mathrm{vp} 0}$ est l'énergie potentielle d'un électron dans le vide au voisinage de la pointe (s'il n'y avait ni abaissement de la barrière de potentiel, ni émission à travers la barrière) et $E_{\mathrm{vg}}$ l'énergie potentielle d'un électron dans le vide au voisinage de la grille. Si l'on admet que le travail de sortie de la pointe et celui de la grille sont sensiblement égaux (disons à $1 \mathrm{eV}$ près), on a :

$$
e V=E_{\mathrm{vp} 0}-E_{\mathrm{vg}} \simeq E_{\mathrm{Fp}}-E_{\mathrm{Fg}},
$$

d'où l'on déduit, en utilisant les relations (3.8) et (3.9) :

$$
V=\left(V_{\mathrm{g}}-V_{\mathrm{s}}\right)-R I_{\mathrm{p}}
$$

C'est à partir de cette relation qu'a été corrigée la courbe en trait plein de la figure 3 , pour donner la courbe en traits interrompus, sur la même figure.

Pour en revenir à la dispersion énergétique, il faut noter que même si la résistance d'interface était rigoureusement la même pour chaque pointe, la présence de cette résistance pourrait affecter la dispersion en énergie, dans la mesure où le courant émis par chaque pointe subit des fluctuations qui se traduisent par une variation de la chute de potentiel au niveau de chaque pointe.

En conclusion de ce paragraphe, on peut dire que cette méthode de retardement ne permet pas d'obtenir une bonne résolution en énergie, du moins avec la structure simple adoptée. En revanche elle permet d'identifier aisément la présence d'une couche d'interface entre les pointes et le substrat, et peut donc servir de test rapide pour la qualité du contact substrat-pointe.

3.2.2 Utilisation d'un spectromètre de bonne résolution. - Pour une mesure précise de la distribution énergétique des électrons, la méthode «évidente " consiste à utiliser un spectromètre d'électrons. $A$ priori, compte tenu des forts courants extraits, l'utilisation d'un spectromètre hémisphérique à retardement (c'est-à-dire un filtre passe-haut) serait la mieux adaptée, car il accepte un courant fort, tout en ayant une précision satisfaisante (environ $0,1 \%$, soit $0,1 \mathrm{eV}$ à $100 \mathrm{eV}$, voir par exemple [18]). Ne disposant pas d'un tel appareil, nous avons utilisé un spectromètre à secteur sphérique à déflection monté sur un appareillage de spectroscopie d'électrons ESCALAB (Vacuum Generators). Ce spectromètre est pourvu d'un diaphragme d'entrée situé entre la source d'électrons à étudier et l'entrée de l'analyseur, qui sélectionne un angle d'acceptance de $\pm 2,5$ 
degrés environ, pour une source située à $75 \mathrm{~mm}$. Cet appareil fonctionne correctement pour des courants faibles $\left(10^{-8} \mathrm{~A}\right)$, avec une résolution meilleure que $50 \mathrm{meV}$. Les mesures ont été réalisées sur une cathode comprenant une seule pointe, puis sur plusieurs cathodes comprenant 10000 pointes déposées sur substrat silicium. Le dispositif expérimental est présenté sur la figure 9.

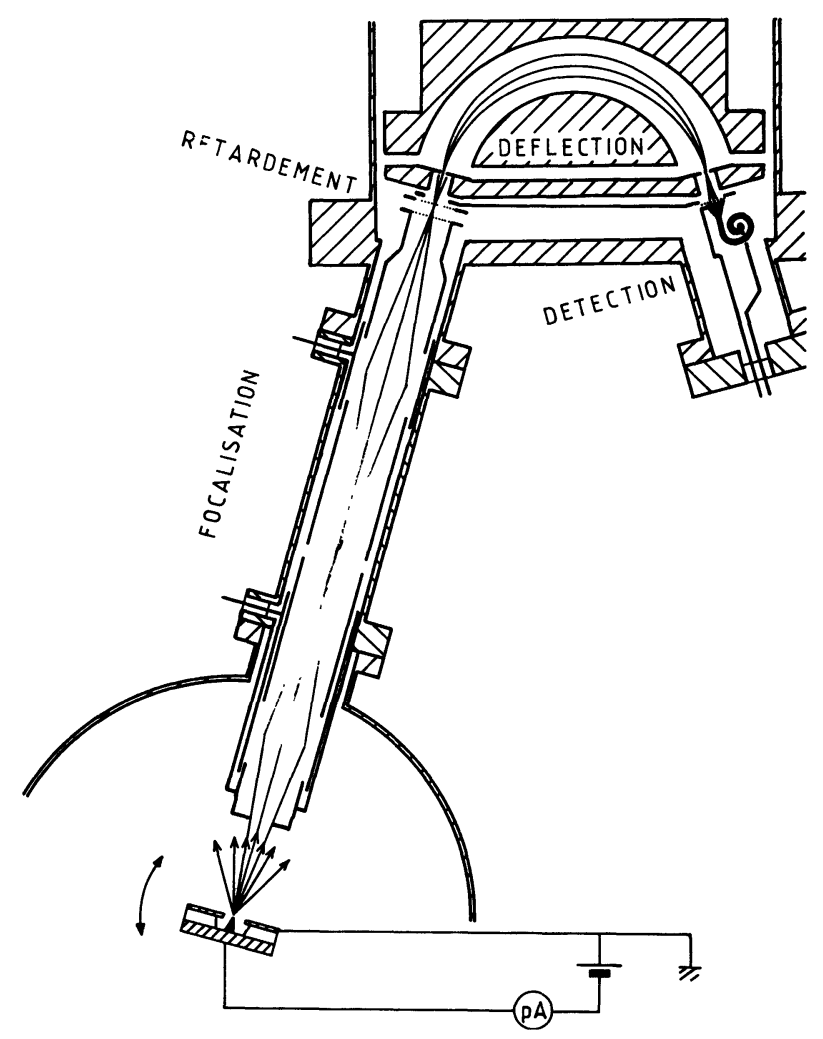

Fig. 9. - Mesure de la distribution énergétique des électrons à l'aide d'un spectromètre à déflection.

[Electron energy distribution measurement, with a deflecting electron spectrometer (schematic).]

La première étude concerne une cathode à pointe unique (cathode $\mathrm{n}^{\circ} 2$ ), délivrant typiquement un courant $1 \mu \mathrm{A}$ pour $V_{\mathrm{g}}-V_{\mathrm{p}}=90 \mathrm{~V}$. Les mesures ont été réalisées à faible tension: $V_{\mathrm{g}}-V_{\mathrm{p}}=27$ à $33 \mathrm{~V}$, ce qui correspond à un courant d'émission très faible (1 à $100 \mathrm{pA})$. Pour ces tensions, le courant subit d'assez fortes fluctuations (environ $50 \%$ ), et surtout la direction d'émission varie très fortement, de sorte qu'au cours du relevé d'un spectre (balayage en énergie), le taux de comptage au niveau du détecteur peut varier brutalement et fortement. Les périodes d'émission stable en direction et en intensité durent de 30 secondes à quelques minutes et alternent avec des périodes d'émission fort instable où les électrons sont émis par «bouffées » qui vont jusqu'à saturer le détecteur. La direction d'émission peut changer brutalement, ce dont on s'aperçoit en réo- rientant la cathode par rapport au spectromètre. Les mesures sont donc extrêmement délicates et les résultats difficilement reproductibles. Lors des périodes d'émission stable, on a pu néanmoins tracer le spectre d'énergie des électrons (voir Fig. 10). La structure observée sur le spectre entre $5 \mathrm{eV}$ et $25 \mathrm{eV}$ correspond à des électrons secondaires créés dans la lentille électrostatique ainsi que dans l'analyseur mais néanmoins détectés. En ce qui concerne le pic d'énergie situé à $32,7 \mathrm{eV}$, la valeur mesurée $\Delta E=0,2 \mathrm{eV}$ est en bon accord avec l'ordre de grandeur calculé au paragraphe 3.1 pour $T=T_{\mathrm{c}}$. Comme le courant est très faible, la résistance d'interface ne joue pas (compte tenu de la résolution du spectromètre), et aucun décalage en énergie n'est observé.

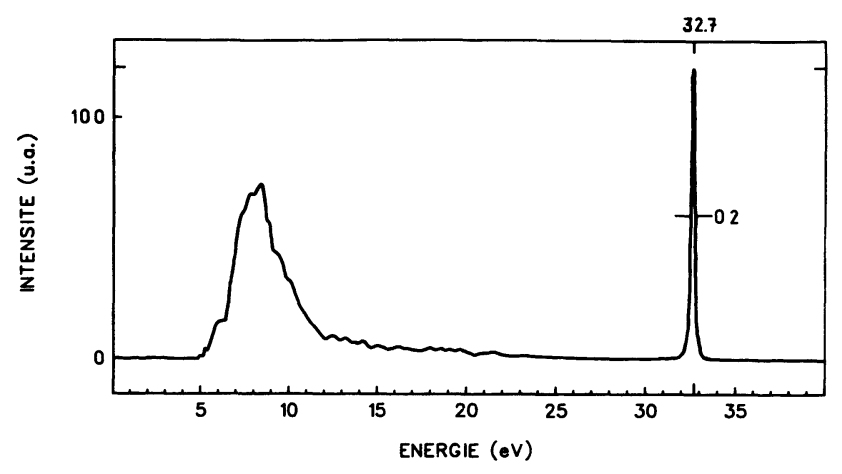

Fig. 10. - Distribution en énergie des électrons émis par une pointe unique (cathode $\mathrm{n}^{\circ} 2$ ). $V_{\mathrm{g}}-V_{\mathrm{p}}=32,7 \mathrm{~V}$.

[Energy distribution of electrons emitted by a single tip cathode (cathode $\mathrm{n}^{\circ} 2$ ). $V_{\mathrm{g}}-V_{\mathrm{p}}=32.7 \mathrm{~V}$.]

Pour une cathode comprenant 10000 pointes en carbure de titane, déposées sur un substrat silicium (cathode $n^{\circ} 3$ ), et faisant partie de la même série que la cathode $\mathrm{n}^{\circ} 1$ étudiée dans les paragraphes précédents, la caractéristique $I_{\mathrm{p}}\left(V_{\mathrm{g}}-V_{\mathrm{s}}\right)$ a été mesurée en trois points: $V_{\mathrm{g}}-V_{\mathrm{s}}=70 \mathrm{~V}, 80 \mathrm{~V}$, et $90 \mathrm{~V}$, donnant des courants $I_{\mathrm{p}}=25 \mu \mathrm{A}, 45 \mu \mathrm{A}$ et $110 \mu \mathrm{A}$. La courbe de Fowler-Nordheim (Fig. 11, trait plein) n'est pas précise, mais on peut en déduire grossièrement le facteur de conversion, et la surface d'émission totale :

$$
\begin{aligned}
& \beta=1,7 \times 10^{8} \mathrm{~m}^{-1}, \\
& S=2 \times 10^{-19} \mathrm{~m}^{2},
\end{aligned}
$$

soit une surface moyenne d'émission par pointe :

$$
S=2 \times 10^{-3} \AA^{2},
$$

ce qui n'est pas acceptable physiquement.

Les mesures de la distribution en énergie correspondant à ces trois points, c'est-à-dire à $e\left(V_{\mathrm{g}}-V_{\mathrm{s}}\right)=E_{\mathrm{Fs}}-E_{\mathrm{Fg}}=70 \mathrm{eV}, 80 \mathrm{eV}$ et $90 \mathrm{eV}$ sont présentées sur la figure 12. L'énergie moyenne 


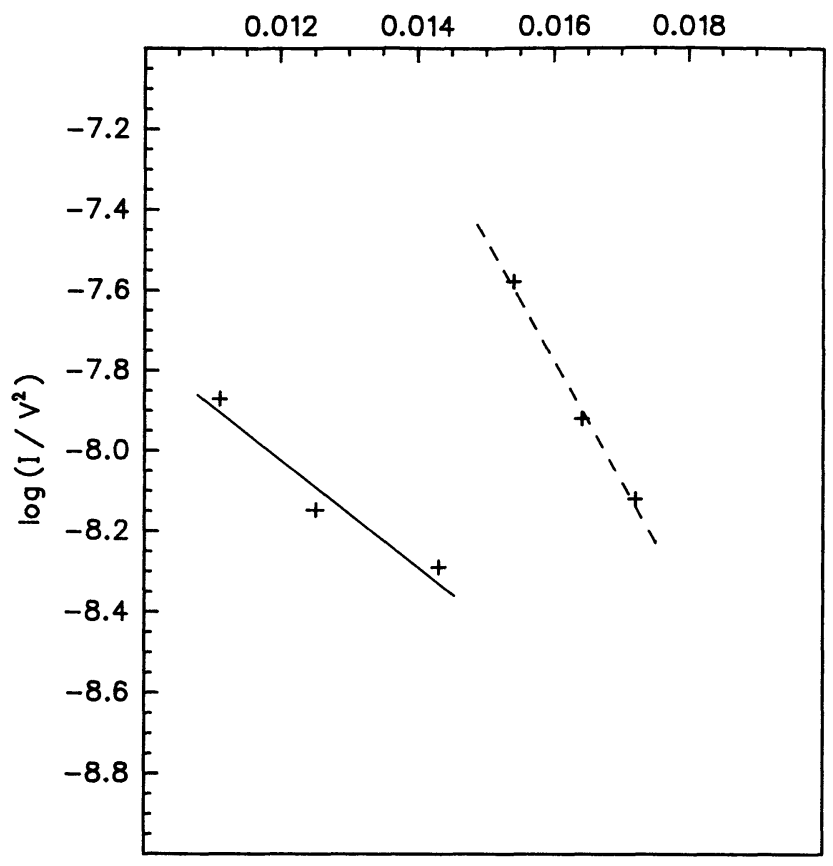

$1 / V\left(V^{-1}\right)$

Fig. 11. - Caractéristique de Fowler-Nordheim d'une cathode à émission de champ (cathode $\left.n^{\circ} 3\right)$. ( non corrigée ; (----) corrigée, en tenant compte de la chute de tension à l'interface.

[Fowler-Nordheim plot of a field emission cathode (cathode $\mathrm{n}^{\circ}$ 3). (— $\longrightarrow$ ) uncorrected ; (----) corrected for the interface voltage drop.]

des électrons, mesurée par rapport au niveau Fermi de la grille vaut respectivement $e V=E_{\mathrm{Fp}}=E_{\mathrm{Fg}}=$ $58 \mathrm{eV}, 61 \mathrm{eV}$ et $65 \mathrm{eV}$, ce qui correspond à une résistance d'interface de $480 \mathrm{k} \Omega, 420 \mathrm{k} \Omega$ et $230 \mathrm{k} \Omega$, si l'on tient compte des valeurs respectives de la tension $V_{\mathrm{g}}-V_{\mathrm{s}}$ et du courant. Les valeurs de la résistance d'interface sont du même ordre de grandeur que celles trouvées pour la cathode $n^{\circ} 1$ étudiée par la méthode du potentiel retardateur. La caractéristique de Fowler-Nordheim, corrigée en tenant compte de ces valeurs, est présentée sur la figure 11 en traits interrompus. Les valeurs du facteur de conversion et de la surface totale d'émission sont alors :

$$
\begin{aligned}
& \beta=7,5 \times 10^{7} \mathrm{~m}^{-1}, \\
& S=4 \times 10^{-15} \mathrm{~m}^{2},
\end{aligned}
$$

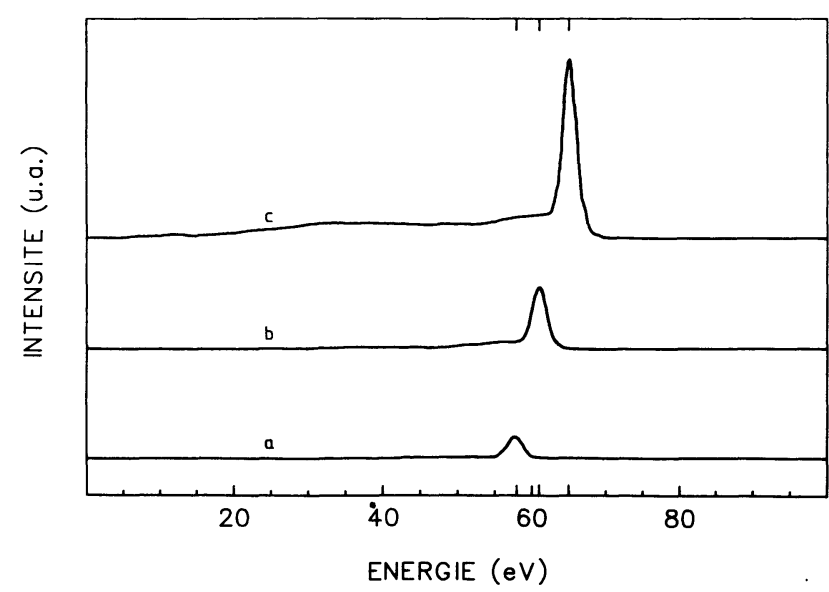

Fig. 12. - Distribution en énergie des électrons émis par une cathode (cathode $\mathrm{n}^{\circ} 3$ ) a) $V_{\mathrm{g}}-V_{\mathrm{s}}=70 \mathrm{~V}$, $I_{\mathrm{p}}=25 \mu \mathrm{A}$; b) $V_{\mathrm{g}}-V_{\mathrm{s}}=80 \mathrm{~V}, \quad I_{\mathrm{p}}=45 \mu \mathrm{A} ; \quad$ c) $V_{\mathrm{g}}-V_{\mathrm{s}}=90 \mathrm{~V}, I_{\mathrm{p}}=110 \mu \mathrm{A}$.

[Energy distribution of electrons emitted by a cathode (cathode $\mathrm{n}^{\circ} 3$ ) a) $V_{\mathrm{g}}-V_{\mathrm{s}}=70 \mathrm{~V}, \quad I_{\mathrm{p}}=25 \mu \mathrm{A}$; b) $V_{\mathrm{g}}-V_{\mathrm{s}}=80 \mathrm{~V}, \quad I_{\mathrm{p}}=45 \mu \mathrm{A} ; \quad$ c) $\quad V_{\mathrm{g}}-V_{\mathrm{s}}=90 \mathrm{~V}$, $\left.I_{\mathrm{p}}=110 \mu \mathrm{A}.\right]$

ce qui correspond à une surface moyenne d'émission par pointe :

$$
s=40 \AA^{2} \text { au lieu de } 2 \times 10^{-3} \AA^{2} .
$$

Ainsi, dans le cas de cette cathode, il ne faut pas négliger la correction de résistance d'interface. Il apparaît donc que le tracé de la caractéristique de Fowler-Nordheim d'une cathode doit s'accompagner de la mesure, même imprécise, de la chute de potentiel à l'interface, sans quoi les résultats concernant la surface d'émission n'ont aucun sens physique.

La connaissance du facteur $\beta$ (corrigé) permet d'évaluer le champ à l'extrémité de la pointe, donc la température critique et la dispersion théorique maximale $\Delta E_{\mathrm{c}}$, par la méthode présentée au paragraphe 3.1. En prenant les valeurs corrigées de $V$ et de $\beta\left(\beta=7,5 \times 10^{7} \mathrm{~m}^{-1}\right)$, et en supposant toujours $\phi=4 \mathrm{eV}$, on obtient les résultats présentés dans le tableau II.

Expérimentalement, on trouve une dispersion énergétique pratiquement indépendante de la tension appliquée, et qui vaut $\Delta E \simeq 2 \mathrm{eV}$. Le fait que

Tableau II. - Dispersion énergétique calculée à $T=0 \mathrm{~K}$ et à $T=T_{\mathrm{c}}$, cathode $\mathrm{n}^{\circ} 3$.

[Energetic spread, calculated for $T=0 \mathrm{~K}$ and $T=T_{\mathrm{c}}$, cathode $\mathrm{n}^{\circ} 3$.]

\begin{tabular}{|c|c|c|c|c|c|c|}
\hline$V_{\mathrm{g}}-V_{\mathrm{s}}(\mathrm{V})$ & $V_{\mathrm{g}}-V_{\mathrm{p}}(\mathrm{V})$ & $E\left(\mathrm{~V} \mathrm{~m} \mathrm{~m}^{-1}\right)$ & $d(\mathrm{eV})$ & $\Delta E_{0}(\mathrm{eV})$ & $T_{\mathrm{c}}(\mathrm{K})$ & $\Delta E_{\mathrm{c}}(\mathrm{eV})$ \\
\hline 70 & 58 & $4,4 \times 10^{9}$ & 0,20 & 0,14 & 1170 & 0,53 \\
80 & 61 & $4,6 \times 10^{9}$ & 0,21 & 0,21 & 1230 & 0,56 \\
90 & 65 & $4,9 \times 10^{9}$ & 0,23 & 0,16 & 1310 & 0,60 \\
\hline
\end{tabular}


pour une seule pointe la dispersion énergétique ait la valeur attendue (lorsque le courant est stable), et que pour 10000 pointes il apparaisse un tel désaccord, incite à penser qu'il y a un effet de "groupe ». Supposons que toutes les pointes aient la même résistance d'interface et qu'elles émettent toutes le même courant. Cela entraîne la même chute de potentiel sur ces pointes (par exemple une chute de potentiel de $25 \mathrm{~V}$ pour $V_{\mathrm{g}}-V_{\mathrm{s}}=90 \mathrm{~V}$ ). Supposons maintenant que le courant émis par une pointe donnée baisse de $5 \%$, cela provoque une diminution de $5 \%$ environ sur la chute de potentiel au niveau de cette pointe, c'est-à-dire que les électrons émis ont à la sortie du dispositif une énergie de $66,2 \mathrm{eV}$ au lieu de $65 \mathrm{eV}$. Si pour une autre pointe, au même moment, le courant augmente de $5 \%$, il en est de même de la chute de potentiel et l'énergie des électrons devient $63,8 \mathrm{eV}$ au lieu de $65 \mathrm{eV}$. Ainsi, en présence d'une chute de potentiel donnée à l'interface, les fluctuations de cette chute de potentiel (d'une pointe à l'autre), sont reliées au fluctuations du courant d'émission d'une pointe au cours du temps. Ce phénomène est atténué par le fait que lorsqu'une pointe émet « trop », la chute de potentiel augmente, ce qui provoque en retour une diminution du courant émis, puisque la tension effectivement appliquée diminue. Plutôt que d'étudier précisément ces fluctuations, il vaut mieux déterminer la résistance maximale tolérable pour qu'elles ne provoquent pas une trop forte dispersion. Par exemple, si l'on se fonde sur une fluctuation de $\pm 50 \%$ sur le courant émis par pointe, la fluctuation maximale de la chute de potentiel est $50 \%$ de cette même chute de potentiel. Ainsi, si l'on parvient à réduire la chute de potentiel à $0,2 \mathrm{eV}$ pour $V=90 \mathrm{~V}$ et $I=100 \mu \mathrm{A}$, la contribution de ce phénomène à la dispersion énergétique sera inférieure à $0,2 \mathrm{eV}$, ce qui requiert (pour 10000 pointes) une résistance d'interface de $2 \mathrm{k} \Omega$ au maximum.

Un premier pas dans cette direction a été franchi en remplaçant le substrat silicium par une couche d'oxyde d'indium. Une dispersion de l'ordre de $1,3 \mathrm{eV}$ pour $I_{\mathrm{p}}=20 \mu \mathrm{A}$ à $60 \mathrm{eV}$ a été mesurée sur une cathode de ce type grâce au spectromètre. A $32 \mathrm{eV}$ cette dispersion ne vaut plus que $0,3 \mathrm{eV}$ pour un courant de $3 \mu \mathrm{A}$.

A ce stade de nos mesures, on pouvait se demander si la «mauvaise » résolution en énergie observée à haute tension n'était pas liée au fait que les électrons analysés étaient issus (grâce à une forte inclinaison de la cathode) de la périphérie du faisceau, où, par suite de chocs inélastiques sur les bords des trous dans la grille, leur énergie aurait pu se dégrader (l'inclinaison de la cathode étant nécessaire pour éviter la saturation du détecteur). C'est la raison pour laquelle nous avons mis en œuvre une troisième méthode dont la précision n'est pas affectée par l'intensité du courant émis.
3.2.3 Utilisation de la photoémission inverse. - Le principe de la photoémission inverse repose sur l'analyse des photons émis lors des transitions radiatives que peuvent subir les électrons pénétrant dans un solide [19]. La figure [13] illustre le diagramme énergétique pour une telle expérience, utilisant comme source d'électrons une multi-cathode à effet de champ et comme échantillon une feuille d'or polycristallin.

Considérons tout d'abord l'émission des électrons et la définition de leur énergie initiale. Comme on l'a vu plus haut, les électrons sont émis au voisinage du niveau de Fermi $E_{\mathrm{Fp}}$ de la pointe, donc leur énergie totale dans le vide est $E_{\mathrm{Fp}}$. Cette énergie se conserve à la traversée de la surface de l'échantillon, par conséquent l'énergie initiale des électrons dans le solide $E_{\mathrm{i}}=E_{\mathrm{Fp}}$. Si $E_{\mathrm{vc}}$ est l'énergie potentielle des électrons dans le vide au voisinage de l'échantillon, et $E_{\mathrm{Fc}}$ le niveau de Fermi de l'échantillon, on peut écrire l'énergie cinétique $E_{\text {cin }}$ des électrons dans le vide au voisinage de l'échantillon et le travail de sortie $\phi_{\mathrm{c}}$ de celui-ci :

$$
\begin{gathered}
E_{\mathrm{cin}}=E_{\mathrm{i}}-E_{\mathrm{vc}}=E_{\mathrm{Fp}}-E_{\mathrm{vc}}, \\
\phi_{\mathrm{c}}=E_{\mathrm{vc}}-E_{\mathrm{Fe}} .
\end{gathered}
$$

La condition pour que les électrons puissent pénétrer dans le solide est que leur énergie cinétique soit positive.

Si $E_{\mathrm{Fs}}$ est le niveau de Fermi du substrat de la cathode, et $V_{\mathrm{e}}-V_{\mathrm{s}}$ la tension à laquelle est porté l'échantillon par rapport à ce substrat, on a :

$$
e\left(V_{\mathrm{c}}-V_{\mathrm{s}}\right)=E_{\mathrm{Fs}}-E_{\mathrm{Fe}},
$$

D'autre part, la chute de potentiel entre le substrat et les pointes est, comme on l'a vu plus haut :

$$
e R I_{\mathrm{p}}=E_{\mathrm{Fs}}-E_{\mathrm{Fp}} \text {. }
$$

Par conséquent, en combinant les équations (3.17) à (3.20), on obtient l'énergie cinétique des électrons :

$$
E_{\mathrm{cin}}=e\left(V_{\mathrm{c}}-V_{\mathrm{s}}-R I_{\mathrm{p}}\right)-\phi_{\mathrm{c}},
$$

avec la condition :

$$
e\left(V_{\mathrm{c}}-V_{\mathrm{s}}\right)>\phi_{\mathrm{c}}+e R I_{\mathrm{p}} .
$$

D'autre part, l'énergie des électrons dans le solide, référencée par rapport au niveau de Fermi de celuici, est :

$$
E_{\mathrm{i}}-E_{\mathrm{Fc}}=e\left(V_{e}-V_{\mathrm{s}}-R I_{\mathrm{p}}\right)
$$

Considérons maintenant les possibilités, pour un électron incident, venu occuper un état d'énergie $E_{\mathrm{i}}$ (initialement inoccupé), de subir une transition radiative. Il ne peut transiter que vers un état inoccupé $\left(E_{\mathrm{f}}\right)$, donc situé au-dessus du niveau de Fermi $E_{\mathrm{Fc}}$ :

$$
E_{\mathrm{Fc}}<E_{\mathrm{f}}\left(<E_{\mathrm{i}}\right)
$$


par conséquent l'énergie du photon émis lors de cette transition est toujours inférieure à une valeur limite $E_{\mathrm{i}}-E_{\mathrm{Fc}}$ :

$$
\hbar \omega<E_{\mathrm{i}}=E_{\mathrm{Fe}}=e\left(V_{\mathrm{e}}-V_{\mathrm{s}}-R I_{\mathrm{p}}\right) .
$$

La probabilité de transition, toujours très faible $\left(10^{-8}\right.$ photon/électron incident. $\left.\mathrm{eV}\right)$ est, pour un état initial donné, proportionnelle à la densité d'états finaux $n\left(E_{\mathrm{f}}\right)$; par conséquent le spectre des photons émis est une image de la densité des états d'énergie inoccupés au-dessus du niveau de Fermi, qui est représentée à droite de la figure 13. Un tel spectre de photons est enregistré à l'aide d'un spectromètre V.U.V. [19] adapté aux expériences où $E_{\mathrm{i}}-E_{\mathrm{Fe}}$ vaut quelques dizaines d'électronvolts. Ce spectromètre est équipé d'une multidétection de photons [20].

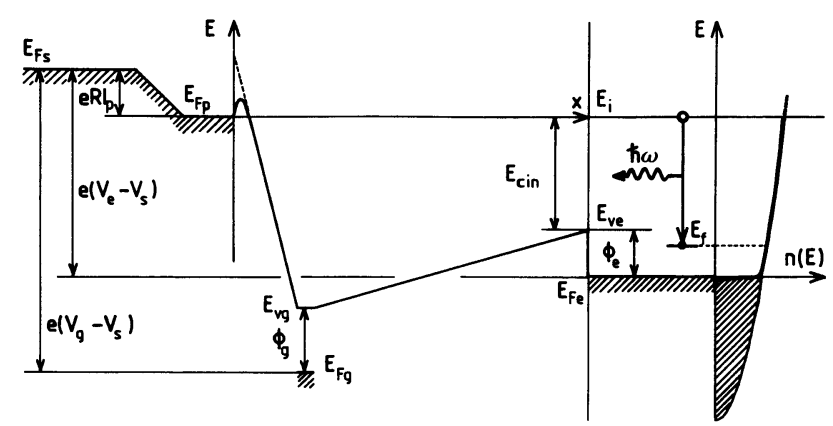

Fig. 13. - Diagramme énergétique d'une expérience de photoémission inverse utilisant une cathode à émission de champ.

[Energy diagram of an inverse photoemission experiment using a field emission cathode.]

A température d'échantillon nulle, pour des électrons émis par la pointe sans dispersion énergétique, et pour un spectromètre de résolution infinie, la cassure au niveau de Fermi (limite inférieure de la distribution en énergie de la densité des états inoccupés $=$ limite supérieure d'énergie des photons) est une marche au-dessus de laquelle le spectre (en énergie des électrons) a une forme parabolique (électrons $\mathrm{s}$ de l'or). Lorsque la température de l'échantillon est d'environ $300 \mathrm{~K}$, il apparaît un élargissement de la marche sur une largeur $2 k T \simeq$ $50 \mathrm{meV}$ que l'on peut négliger ici. Le spectromètre, quant à lui, a une résolution finie et connue $\Delta \lambda$, ce qui entraîne un élargissement en énergie $\Delta E_{\text {spec }}$ sur le spectre, calculable à partir de la relation:

$$
E(\mathrm{eV})=\frac{12400}{\lambda(\AA)},
$$

d'où :

$$
\Delta E_{\mathrm{spcc}}(\mathrm{eV})=\frac{\Delta \lambda(\AA) E^{2}(\mathrm{eV})}{12400}
$$

Enfin la dispersion en énergie des électrons émis par la pointe, $\Delta E_{\mathrm{el}}$, se traduit par un élargissement supplémentaire de la marche. Si l'on admet que les deux principales sources d'élargissement correspondent à des gaussiennes, l'élargissement total vaut :

$$
\Delta E=\left(\left(\Delta E_{\mathrm{el}}\right)^{2}+\left(\Delta E_{\mathrm{spec}}\right)^{2}\right)^{1 / 2}
$$

correspondant à la montée de la marche entre $15 \%$ et $85 \%$. Ainsi, en mesurant $\Delta E$, on en déduit $\Delta E_{\mathrm{cl}}$, pourvu que $\Delta E_{\text {spec }}$ ne soit pas trop fort.

L'expérience a été menée pour une cathode comprenant 10000 pointes en niobium déposées sur un substrat en oxyde d'indium (cathode $n^{\circ} 4$ ), l'échantillon étant une feuille d'or. La tension appliquée sur la grille est $V_{\mathrm{g}}-V_{\mathrm{s}}=59 \mathrm{~V}$; le courant maximum émis $I_{\mathrm{p}}$ vaut environ $100 \mu \mathrm{A}$ (mesuré en appliquant une tension $V_{\mathrm{e}}-V_{\mathrm{s}}$ de quelques centaines de volts sur l'échantillon). Lors de l'enregistrement du spectre, la tension appliquée sur l'échantillon est $V_{\mathrm{e}}-V_{\mathrm{s}}=25 \mathrm{~V}$, et le courant recueilli par l'échantillon n'est plus que de $55 \mu \mathrm{A}$. Le spectre obtenu est représenté sur la figure [14], où l'on a reporté le nombre de photons émis en fonction de l'énergie. Le milieu de la montée de la marche représente la position énergétique du niveau de Fermi de l'échantillon, et correspond à une énergie de photons $\hbar \omega=23,7 \mathrm{eV}$.

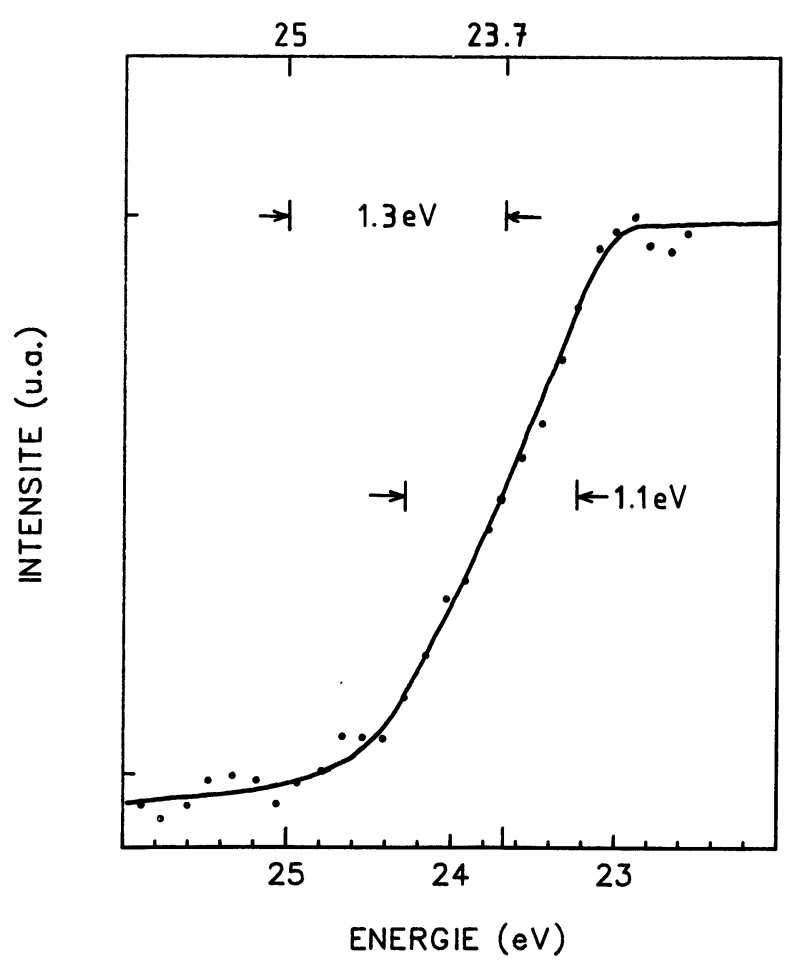

Fig. 14. - Spectre de photoémission inverse de l'or polycristallin. La source d'électrons est une cathode à émission de champ (énergie : $23,7 \mathrm{eV}$, courant $55 \mu \mathrm{A}$ ).

[Inverse photoemission spectrum of polycrystalline gold. The electron source is a field emission cathode (energy : $23.7 \mathrm{eV}$, current $55 \mu \mathrm{A})$.] 
D'après (3.25) on a :

$$
\hbar \omega=e\left(V_{\mathrm{e}}-V_{\mathrm{s}}-R I_{\mathrm{p}}\right),
$$

soit en volts :

$$
23,7=25-R I_{\mathrm{p}},
$$

d'où une chute de tension :

$$
R I_{\mathrm{p}}=1,3 \mathrm{~V},
$$

et une résistance (en prenant $I_{\mathrm{p}}=100 \mu \mathrm{A}$ ) :

$$
R=13 \mathrm{k} \Omega\left(\text { pour } V_{\mathrm{g}}-V_{\mathrm{s}}=59 \mathrm{~V}\right) .
$$

Cette valeur est intermédiaire entre les résultats obtenus avec un substrat silicium $(R$ de l'ordre de $100 \mathrm{k} \Omega)$, et l'objectif fixé $(R=2 \mathrm{k} \Omega)$ qui garantirait que l'influence de la résistance d'interface ne se traduise pas par une dispersion de plus de $0,2 \mathrm{eV}$.

La largeur de la montée entre $15 \%$ et $85 \%$ est environ :

$$
\Delta E \simeq 1,1 \mathrm{eV} .
$$

Comme la résolution en longueur d'onde du spectromètre est de $13 \AA$ environ, cela donne pour une énergie de $23,7 \mathrm{eV}$, une résolution en énergie du spectromètre :

$$
\Delta E_{\mathrm{spcc}}=0,6 \mathrm{eV} .
$$

On en déduit, à l'aide de la relation (3.28), la dispersion énergétique des électrons émis :

$$
\Delta E_{\mathrm{cl}}=0,9 \mathrm{eV} \text {. }
$$

La caractéristique de Fowler-Nordheim de cette cathode a été tracée entre $40 \mathrm{~V}$ et $50 \mathrm{~V}$ (compte non tenu de la correction liée à la chute de potentiel d'interface qui est faible dans ce cas). On obtient $\beta=1,8 \times 10^{8} \mathrm{~m}^{-1}$. Les fluctuations d'émission ayant rendu les mesures difficiles, cette valeur de $\beta$ est sujette à caution. Toutefois une estimation de la dispersion théorique attendue peut être faite : pour $V_{\mathrm{g}}-V_{\mathrm{s}}=59 \mathrm{~V}$ on a : $V=57,7 \mathrm{~V}$, le champ vaut $\|\boldsymbol{E}\|=1.1 \times 10^{10} \mathrm{Vm}^{-1}$, la dispersion à $0 \mathrm{~K}$ est $\Delta E_{0}=0,34 \mathrm{eV}$, la température critique est $T_{\mathrm{c}}=$ $2800 \mathrm{~K}$, et à cette température, la dispersion en énergie vaut $\Delta E_{\mathrm{c}}=1,3 \mathrm{eV}$. La valeur mesurée de la dispersion se situe dans cette fourchette, mais il faut garder présent à l'esprit le fait que les valeurs théoriques sont peu précises pour cette cathode car le courant d'émission fluctue assez fortement lors du relevé de la caractéristique.

La méthode proposée dans ce paragraphe permet donc de caractériser la dispersion en énergie et le décalage dû à la chute de potentiel à l'interface, sur une expérience au cours de laquelle la plus grande fraction du courant émis est testée. Les pointes déposées sur un substrat en oxyde d'indium représentent une amélioration par rapport aux cathodes déposées sur substrat silicium, du point de vue de la résistance d'interface. D'autre part, la dispersion énergétique est aussi plus faible, ce qui semble indiquer une corrélation entre la chute de potentiel à l'interface et la dispersion, comme cela était suggéré dans le paragraphe précédent.

\section{Conclusion.}

L'utilisation des cathodes à émission de champ de conception classique (c'est-à-dire à pointe unique) est actuellement limitée aux expériences où l'énergie finale des électrons est relativement élevée (plus de $200 \mathrm{eV}$ ) et où le courant final du faisceau est faible (moins de $10 \mu \mathrm{A}$ ). Cette situation est principalement due aux contraintes technologiques concernant la distance pointe-anode. Le principal avantage de ces sources est leur forte brillance, qui permet d'obtenir des spots de forte densité de courant et de faible dimension. Les conditions de fonctionnement de la pointe émissive sont bien contrôlées grâce aux procédures de formation sous champ et les méthodes de caractérisation sont maintenant connues (microscopie ionique par effet de champ, analyse de la distribution énergétique des électrons émis...).

En ce qui concerne les cathodes à micropointes, qui permettent d'obtenir des courants forts à faible énergie, l'état de l'art actuel est nettement en retrait, puisque d'une part la technologie adaptée ne s'est développée que récemment, et que d'autre part les méthodes de caractérisation de la distribution énergétique des électrons émis, ainsi que les méthodes de conditionnement des surfaces émissives sont des sujets encore peu explorés. Les possibilités offertes par ce type de sources d'électrons sont d'ores et déjà intéressantes à faible énergie puisque les courants accessibles sont de l'ordre de $100 \mu \mathrm{A}$ par $\mathrm{mm}^{2}$ à $60 \mathrm{eV}$, pour une dispersion en énergie voisine de $0,9 \mathrm{eV}$. Rien ne s'oppose à ce qu'on obtienne, pour la même caractéristique d'émission, une dispersion de l'ordre de $0,3 \mathrm{eV}$, grâce à l'abaissement des résistances d'interface, ce qui est possible par le choix du matériau supportant les pointes. A cet égard notre conclusion diffère de celle qui est exprimée par les auteurs de la référence [6], fondée sur une valeur de la dispersion $\Delta E \simeq 1,9 \mathrm{eV}$ pour un faisceau de courant $0,6 \mu \mathrm{A}$ et d'énergie $150 \mathrm{eV}$.

Les améliorations possibles font envisager l'emploi de ces cathodes dans de nombreux dispositifs où la résolution en énergie et l'intensité de courant à faible énergie sont des paramètres importants. L'objectif d'une résolution ultime de $0,1 \mathrm{eV}$ et d'une intégration plus poussée (100 000 pointes par $\mathrm{mm}^{2}$ ) ne semble pas déraisonnable avec les technologies dont on dispose. Enfin, comme nous l'avons vérifié, l'intensité et la stabilité du courant émis par ces cathodes ne sont pas affectées par un fonctionnement à basse température $(T<100 \mathrm{~K})$, d'où la possibilité de disposer de sources d'électrons froides non polluantes et ne rayonnant pas de chaleur. 


\section{Bibliographie}

[1] Fowler, R. H. and Nordheim, L. W., Proc. $R$. Soc. London A 119 (1928) 173.

[2] Denizart, M., Thèse, Université de Toulouse, 1981.

[3] Spindt, C. A., Brodie, I., Humphrey, L. and Westerberg, E. R., J. Appl. Phys. 47 (1976) 5248.

[4] Meyer, R., Ghis, A., Rambaud, P. and Muller, F., Jpn Displays 86 (Society for Informatic Display) 1986.

[5] Curtis, C. C. and Hsieh, K. C., Rev. Sci. Instrum. 57 (1986) 989.

[6] Mitchell, R. E., Mitchell, J. B. A. and McGoWAN, J. W., J. Phys E 18 (1985) 1031.

[7] Young, R. D., Phys. Rev. 113 (1959) 110.

[8] Gadzuk, J. W. and Plummer, E. W., Rev. Mod. Phys. 45 (1973) 487.

[9] Charbonnier, F. M. and Martin, E. E., J. Appl. Phys. 33 (1961) 1897.

[10] Muller, F., Mémoire C.N.A.M., Paris, 1986.

[11] Tomazschke, H. and Alpert, D., J. Appl. Phys. 38 (1967) 881.
[12] Duke, C. B. and Alferieff, M. E., J. Chem. Phys. 46 (1967) 923.

[13] Bell, A. E. and Swanson, L. W., Phys. Rev. B 19 (1979) 3353.

[14] Swanson, L. W. and Crouser, L. C., Phys. Rev. 163 (1967) 622.

[15] Levine, P. H., J. Appl. Phys. 33 (1962) 582.

[16] Charbonnier, F. M., Strayer, R. W., Swanson, L. W. and Martin, E. E., Phys. Rev. Lett. 13 (1964) 397.

[17] El-Kareh, A. B., Wolfe, J. C. and Wolfe, J. E., J. Appl. Phys. 48 (1977) 4799.

[18] Roy, D. and CARETTE, J. D., in Electron Spectroscopy for Surface Analysis, Ed H. IBACH (Springer Verlag, Berlin) 1977.

[19] Chauvet, G. and Baptist, R., J. Electron Spectrosc. Relat. Phenom. 24 (1981) 255.

[20] Chauvet; G., Brenac, A. and Baptist, R., Rev. Sci. Instrum. 58 (1987) 197. 